Chapter 4

\title{
Ischemic Mitral Regurgitation: From Echo Assessment to Surgical Strategy and Techniques
}

\author{
Gheorghe Cerin, Adrian Bogdan Popa, \\ Razvan Ticulescu and Marco Diena \\ Additional information is available at the end of the chapter \\ http://dx.doi.org/10.5772/56062
}

\section{Introduction}

Ischemic mitral regurgitation remains an underestimated and important clinical problem. It is a complex multifactorial disease that involves global and regional left ventricular remodeling as well as dysfunction and distortion of the components of the mitral valve including the chordae, annulus and leaflets. The prevalence rate of development of mild or more severe degree of mitral insufficiency after myocardial infarction has been estimated to be up to $50 \%$ and is associated with worse prognosis (Stevenson 1987, Lamas 1997, Grigioni 2001).

Based on the clinical presentation, the ischemic mitral regurgitation was classified as acute or chronic (Mitesh, 2009). Although the acute ischemic mitral regurgitation is relatively rare, whenever present, it has a rapid evolution, with an extremely poor survival rate. On the other hand, the chronic ischemic mitral regurgitation is much more frequent, but it's the consequence of a slower pathological process, with a better survival rate. The echocardiography identifies all patterns of ischemic mitral regurgitation; nonetheless the classification as acute, sub-acute or chronic ischemic mitral regurgitation is rather clinical, than an echocardiographic one. Beside the complete rupture of papillary muscle, other patterns of ischemic mitral regurgitation cannot be practically located in time by echo. It is possible to have the same echocardiographic feature of the lesion in acute and in chronic settings; only the clinical presentation of the patient helps in the diagnosis of acute / sub-acute or chronic ischemic mitral regurgitation. So the echo exam has to be seen as complementary to clinical data, rather than a singular method of diagnosis.

In everyday practice the management of ischemic mitral regurgitation represents a combined problem of physiopathology, quantification and diagnosis that, together, drive the individual 
patient prognosis but, in case of patients candidate to open heart surgery this becomes a very complex issue. With intraoperative TEE echocardiography, the exact identification of the mechanism of ischemic mitral regurgitation represents a cornerstone element for surgical planning and patient management. In case of significant residual mitral regurgitation a second run of the extracorporeal circulation may be necessary requiring a well-founded experience from the echocardiographer as the TEE intraoperative findings may be sometimes misleading.

The relation between the ischemic mitral regurgitation, surgery and echocardiography, as tool for surgical planning and postoperative control, will be addressed in this chapter. The suboptimal results obtained by the most commonly used surgical strategy, that is, restrictive annuloplasty combined with coronary artery bypass graft, emphasizes the need to develop alternative or concomitant surgical techniques that directly target the causal mechanisms of the disease. Recent data show that this procedure is associated with a $10 \%$ to $20 \%$ rate of persistent mitral regurgitation soon after operation and a 50\% to $70 \%$ rate of recurrent mitral insufficiency at five years (Hashim 2012, Magne 2009). Hereafter, the topics will be focused mainly on the echo assessment of the patients candidate for open-heart surgery. A particular attention will be paid to the papillary muscle and to the mitral valve geometry and their role in the pathophysiology of ischemic mitral insufficiency. Understanding the mechanisms of the different types of ischemic mitral regurgitation is mandatory for echocardiographer and surgeon to tailor the right strategy for the valve repair, and it is indeed a challenging task for both.

\section{Physiopathology: Concepts regarding post-myocardial left ventricular remodeling, acute and chronic ischemic mitral valve and the valve geometry}

It is generally assumed that the ischemic mitral regurgitation is not a valve disease, the 'engine' of valve insufficiency being the remodeling process of the left ventricle, depending on the localization and extension of the myocardial infarction. In ischemic heart disease, the mitral leaflets (and also the chordae) are typically structurally normal, but the leaflets are tethered and their motion is relatively restricted, owing to regional and global ventricular remodeling, with apical displacement of the posteromedial papillary muscle.

Sometimes the remodeling process starts immediately after the onset of myocardial infarction causing structural ischemic mitral regurgitation, which is associated with a poor prognosis, due to the concomitant acute infarction, acute papillary muscle remodeling and acute ischemic mitral regurgitation. For a successful management of this acute mechanical complication the key point is the early diagnosis, which has to be done before the complete rupture of the papillary muscle occurs. This life-threatening complication has otherwise to be considered a failure due to late echocardiographic and clinic diagnosis.

Overall, there is only scanty literature concerning the echocardiographic assessment of the papillary muscle and it is no surprising then to see that its use in the everyday practice is, so 
far, very limited. There are only few general recommendations and the guidelines do not include them in the systematic evaluation of the ischemic mitral regurgitation. Most of the current information focuses on regional and global ventricular remodeling, with leaflets tethering and tenting, motion restriction and annular dilatation. Frequently, the ischemic remodeling of papillary muscle is difficult to document; nevertheless with an accurate analysis, often performed in non-standard echocardiographic views, the diseased papillary muscle may be identified.

In coronary patients with mitral insufficiency, beside the ventricular remodeling, annular dilatation and leaflets (mobility and tethering), the echocardiographer has to focus the attention on the shape, the length and the thickness of the papillary muscle, mainly in acute circumstances, when the acute remodeling process is just beginning (fig 1).
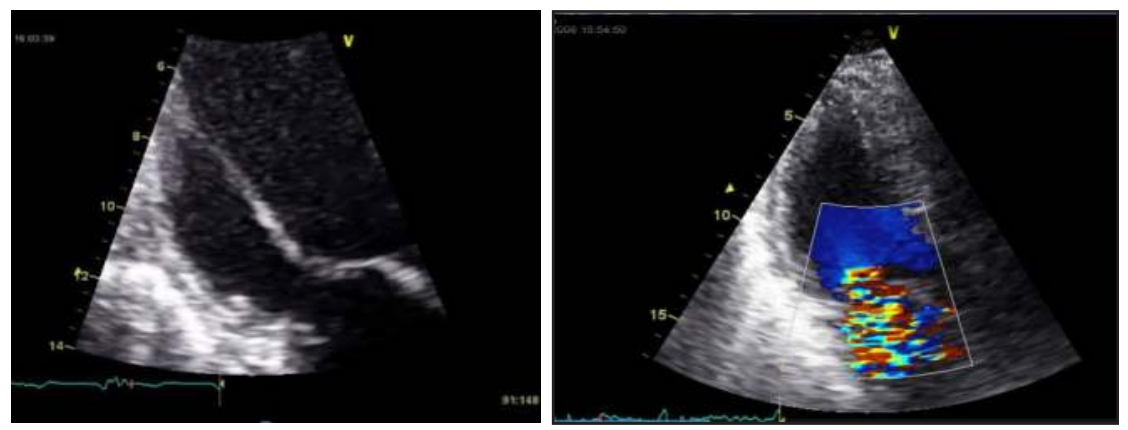

Figure 1. Acute inferior MI with severe MR due to acute post-ischemic elongation of PP MM. Note the abnormal length and shape of the very slim PP MM. The tip reaches the level of the MV annular plane. Often (as in this case), the elongation PP MM is visible in a different plane respect of the MR at color Doppler. $\mathrm{MI}=$ myocardial infarction; $\mathrm{MR}=$ Mitral regurgitation; $\mathrm{PP} M \mathrm{MM}=$ papillary muscle; $\mathrm{MV}=$ mitral valve.

On the other hand, in the case of chronic ischemic mitral insufficiency, all the remodeling processes of left ventricle causing the ischemic mitral regurgitation are slower, the evolve over time and usually the echo diagnosis is not so tricky. Generally, the patterns of chronic ischemic mitral regurgitation are quite often the same: tethering of the papillary muscle, chordae and leaflets, various degree and progressive dilation of the left ventricular cavity and mitral annulus, mitral valve tenting, with reduced / loss of coaptation and restriction of one or both leaflets (fig 2,3). The post-infarction loss of the contractile ventricular mass is directly correlated with the remodeling process, expressed by the heart dilatation and decreasing of cardiac reserve. All the elements belonging to the mitral apparatus will be involved in this process, but the leaflets and the chordae are, as a matter of fact, preserved.

Ischemic mitral regurgitation has several pathophysiological determinants. It has been defined as a ventricular disease that affects the mitral apparatus, because both myocardial infarction and ischemia generate progressive global and regional left ventricular remodeling, and consequently, a functional mitral regurgitation. Apical and posterior displacement of the papillary muscle is a typical echocardiographic finding. This process starts ordinarily with the 

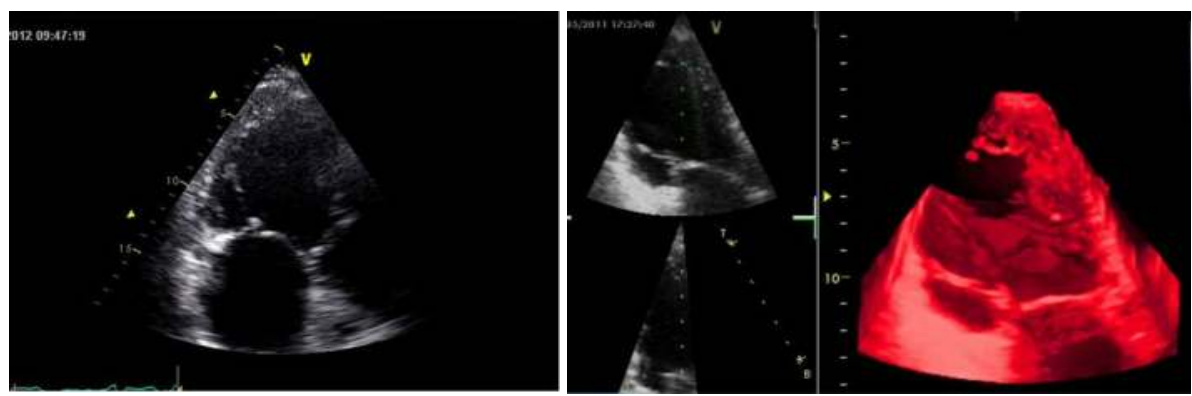

Figure 2. Inferior myocardial infarction with ischemic mitral regurgitation due to systolic restriction of posterior mitral leaflet (2D and 3D systolic frame, from two different patients). Note the systolic tension on the chordae belonging to the posterior mitral valve with limited excursion of the cusp, the asymmetrical apposition of the anterior leaflet and the reduced area of coaptation.

posterior leaflet because the posterior mitral annulus is thinner, weaker, more flexible and without a zone of resistance. On the contrary, the anterior leaflet has a more thickened and less malleable annulus. In this way the posterior leaflet became firstly restricted while the anterior is involved only later on. The echocardiography is able to assess the relationship between the LV cavity (shape, dimensions, volume), the mitral leaflets (as mobility, leaflet apposition, length and height of coaptation, annulus dimension, etc) and the valve geometry.

In the case of chronic ischemic mitral regurgitation and ischemic dilated cardiomyopathy, (generally patients with anterior and inferior infarction), the right balance between the dimension of the mitral valve and the 'new' dimension of the double infarcted ventricular cavity is lost, so that the mitral valve becomes insufficient. Conceptually, it may be assumed that there is a phenomenon of 'mismatch' between the 'too large' left ventricle and 'too small' mitral valve apparatus, which is unable to extend the drapery of the leaflets and to adapt to thenew dimension of the infarcted ventricle (fig 3). That's why, in case of critical coronary artery disease leading to left heart dilatation and ischemic mitral regurgitation, the 'standard' surgical approach of coronary artery by-pass grafting (CABG) plus undersized ring alone, may not solve completely the mitral regurgitation and, over time, the repair may fail (Magne 2009). In these cases the annuloplasty ring alone is not enough to achieve sufficient 'fabrics' for a stable coaptation and repair. Ideally speaking, to get a good repair more tissue is needed to enlarge the mitral valve. This concept will be addressed in the chapter of chronic ischemic mitral regurgitation.

The chronic ischemic mitral regurgitation is classified as a $I I I^{\circ} b$ type of mitral insufficiency essentially due to the systolic restriction of the posterior leaflet (Table 1). The practice demonstrates that in the case of ischemic mitral regurgitation this classification may be incomplete and misleading because the chronic ischemic mitral regurgitation has always a combined annular dilation of the valve, which in fact is a type $\mathrm{I}^{\circ}$ mechanism of mitral regurgitation in Carpenter's classification (Carpentier 1983 \& 2010). That's why, for a better and more precise diagnosis of the mechanisms of ischemic mitral regurgitation, the mitral valve geometry has to be carefully assessed. 


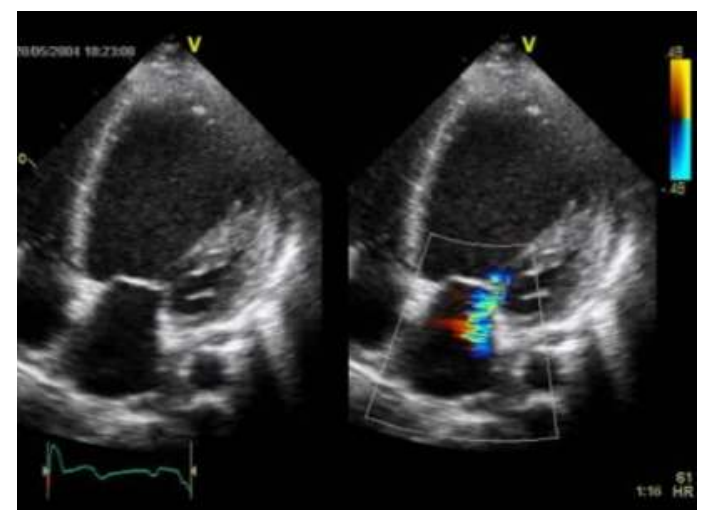

Figure 3. Ischemic dilated cardiomyopathy and the concept of "mitral - ventricular mismatch" (see the text). Patient with double myocardial infarction (anterior and inferior).Ischemic dilated cardiomyopathy and chronic ischemic mitral insufficiency. Note the spherical shape and dilatation of the left ventricular cavity, the reduced surface of coaptation of mitral leaflet $(2 \mathrm{~mm})$, due to the annular dilatation $(41 \mathrm{~mm})$ and the laterally migration of the papillary muscle.

\begin{tabular}{ll}
\hline Type ${ }^{\circ}$ & Normal leaflet motion \\
\hline Type $I^{\circ}$ & Excess leaflet motion (leaflet prolapse) \\
\hline Type III & Restricted motion \\
\hline$I I^{\circ}{ }_{\mathrm{a}}$ & Restricted opening \\
\hline $\mathrm{II}_{\mathrm{b}}$ & Restricted closure \\
\hline
\end{tabular}

Table 1. Carpentier's classifications of Mitral Regurgitation

As noted (Cerin 2006, Tesler 2009), from the geometrical point of view, the normal mitral valve presents a triangle delimited between the coaptation point (typically sited inside of the ventricular cavity) and two others referral points, placed on the anterior and posterior mitral annulus (standard pattern of a normal mitral valve - Fig 4). This echocardiographic virtual tool demonstrates to be useful in judging the mitral valve structural and functional abnormalities (Cerin 2012). When the echocardiographic presentation of ischemic mitral regurgitation is consistent with obvious deformation of this virtual triangle, this should raise the suspicion of a possible papillary muscle abnormality with elongation of sub-valvular apparatus, alongside to reduce / loss of leaflet coaptation.

In some cases the geometry of the mitral valve may be so distorted that an elongation of the sub valvular apparatus has to be suspected or is clearly present. In these particular cases, the echocardiography examination has to be focused on the papillary muscle lesions; this might require the execution of the exam in several off axis views, beside the standard ones. Patients with distortion of mitral valve geometry (fig 5A) will not fit within the III ${ }^{\circ} \mathrm{b}$ of Carpentier's classification. Some of these patient may present a clear ischemic mitral valve prolapse (type $\mathrm{II}^{\circ}$ Carpentier), but many of them will show only a distortion of the 'standard' mitral valve triangle, with the coaptation point still present inside of the left ventricular cavity. The 

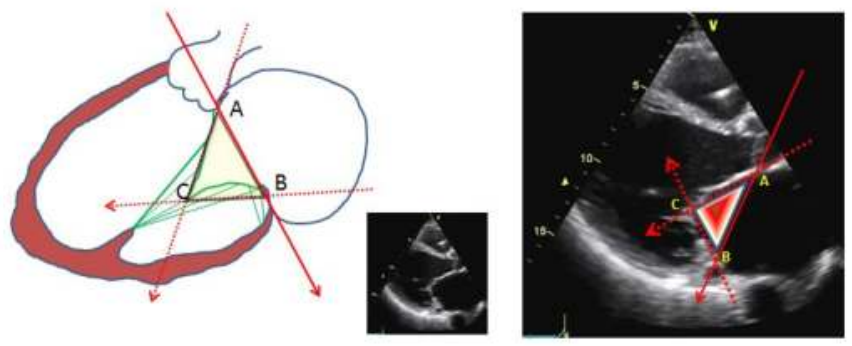

Figure 4. The coaptation triangle and the mitral valve geometry. The normal pattern of mitral valve anatomy and function is characterized by the triangle of coaptation which is defined by the coaptation point (C), normally sited inside of the left ventricular cavity and two other points $(A, B)$ placed on the anterior and posterior mitral annulus.

geometrical analysis of these patients will show the disappearance of the mitral valve triangle and a progressive the transformation of it in a 'trapeze'. (Fig 5B).
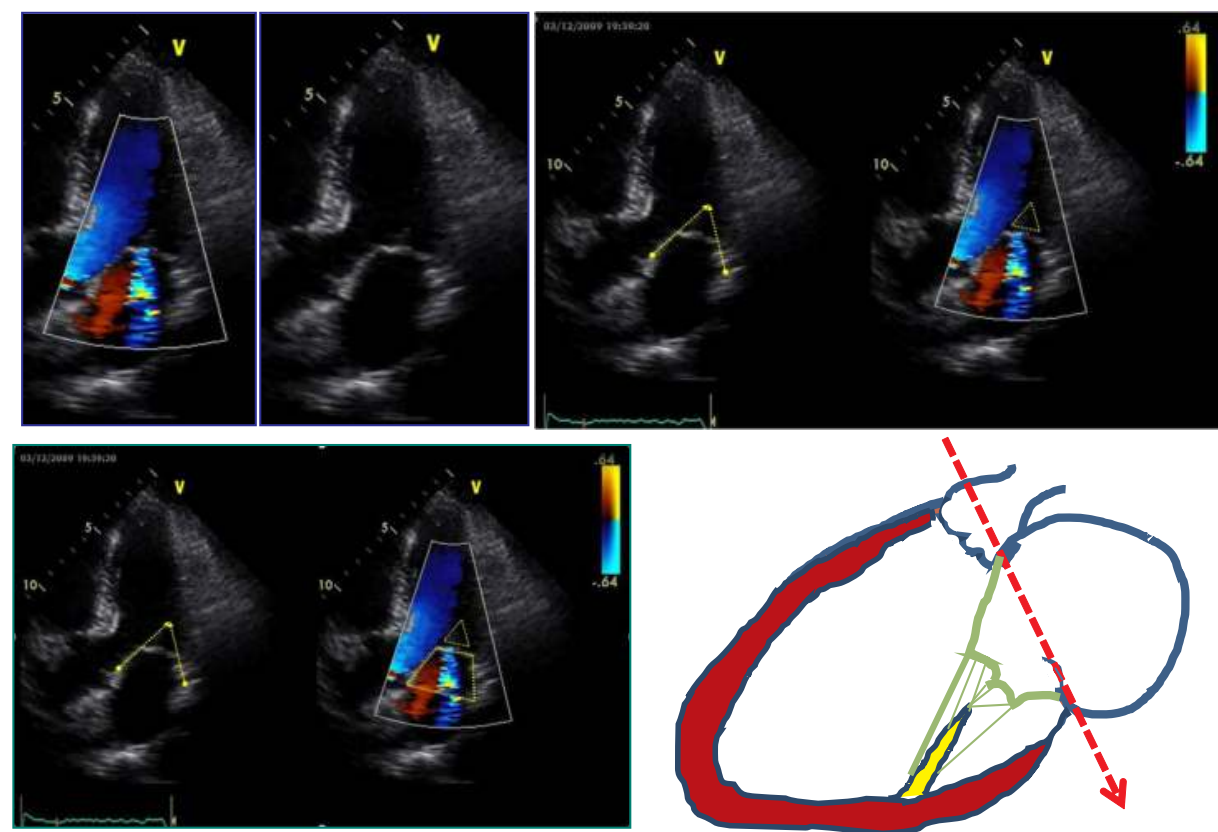

Figure 5. A (up) and B (down). Ischemic mitral regurgitation with distorted mitral valve geometry. Note the absence of the 'standard triangle of coaptation' which as a matter of fact was transformed into a 'trapeze' due to the postischemic 'crash' of the edge of the mitral valve, generated by papillary muscle elongation (drawing).

The repair strategy by the undersized rings alone may not solve the mitral regurgitation in patients with distorted mitral valve geometry (due to papillary muscle elongation). This 
because the small ring corrects the mitral annulus deformation only, whereas the main problems of these patients are represented by the displacement of coaptation plane too 'high', nearby the mitral annulus, the distortion of the coaptation surface and plane due to the 'crash' of the tip of the mitral valve tenting and the reduced mitral leaflet coaptation (fig 5B, scheme). The mechanism of regurgitation in these patients is clearly more complex than the classical 'leaflet restriction' (III’b type of Carpentier's classification).

Often the mechanisms involved in the ischemic mitral regurgitation are multiple, complex and intricate, and the surgical planning of the valve repair must to be tailored, ignoring partially and sometimes totally the classifications; classifications often focus on the mitral leaflet only (which are practically normal) and do not include the papillary muscles, the mitral valve geometry and the grade of general or regional left ventricular remodeling.

Depending of the clinical context, a check list of the echocardiographic elements has to include: dimension of the left ventricular cavity (as diameter and volume), diameter of the mitral annulus (transthoracic, PSLAx systole), the sphericity index and the distance between the papillary muscle (in case of ischemic cardiomyopathy), the length of leaflets coaptation, the length of the anterior leaflet, the 'height' of the posterior leaflet, the mobility of the anterior and most of all the posterior leaflet, etc.

From this point of view, beside of the others elements involved in valve regurgitation, the use of the triangle of coaptation as tool of mitral valve geometry (Cerin 2010) shows to be a suitable instrument 'to judge' better the mechanisms of ischemic mitral regurgitation (Fig 4, 5A, 5B). In some cases, due to obviously papillary muscle elongation, the triangle may completely disappear (see the Fig 6).
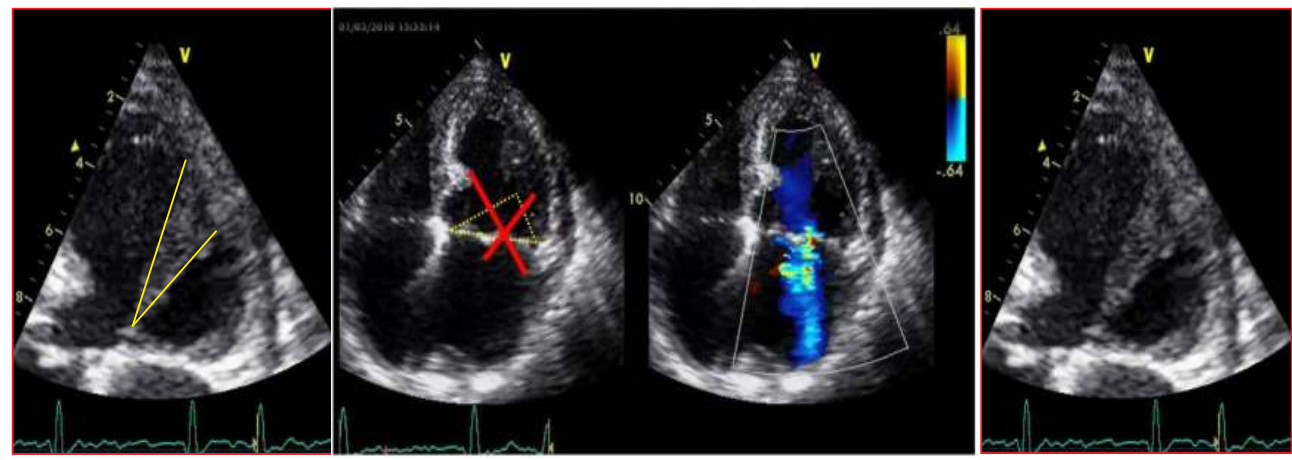

Figure 6. Chronic ischemic mitral regurgitation, due to reduce leaflet coaptation (approx. $2 \mathrm{~mm}$ ), dilatation of the left ventricle (EDVol $245 \mathrm{ml}$ ) and of the mitral annulus $(\varnothing 45 \mathrm{~mm})$. Note the absence of 'the coaptation triangle' due to ischemic elongation of papillary muscle: the length of papillary muscle is completely unusual, having the tip situated very near to the mitral annulus. Note also the triangular shape of this muscle, with a very sharp tip, due to progressive post-ischemic remodeling with elongation: so called 'thorn papillary muscle'. Because of the abnormal post-ischemic elongation of the papillary muscle, the normal geometry of mitral valve was distorted and the triangle of coaptation disappears. NB - all the images belong to the same patient. 
The echocardiographic assessment of the mechanisms of the ischemic mitral regurgitation, which is the key for the tailored surgical approach, may be sometimes complex and tricky, due to a true mosaic of intricate mechanisms, difficult to be embedded and defined by a standard classification. The echocardiographer has to be aware of the complexity of the left ventricular remodeling process and to assess the mitral valve apparatus using multiple views and to pay particular attention to the analysis of the mitral valve geometry.

\section{Acute ischemic mitral regurgitation}

Background. Acute ischemic mitral valve regurgitation may occur in the setting of acute myocardial infarction owing to necrosis and rupture of papillary muscle tissue or abnormal coaptation of the leaflets, due to distortion of ventricular geometry. Hemodynamically significant mitral regurgitation is possible in both anterior and inferior infarcts, but its incidence appears higher in the inferior myocardial infarction group such as $40 \%$ vs $15 \%$ - see the Fig 7 (Estes 1966, Dagum 2000, Timek 2003). Post-infarction mitral regurgitation may be associated to multiple cardiovascular risk factors such as diabetes, abnormal body mass index $(>25)$, advanced age, female gender, coexistence of peripheral arteriopathy and prior infarction (Green, 1999).
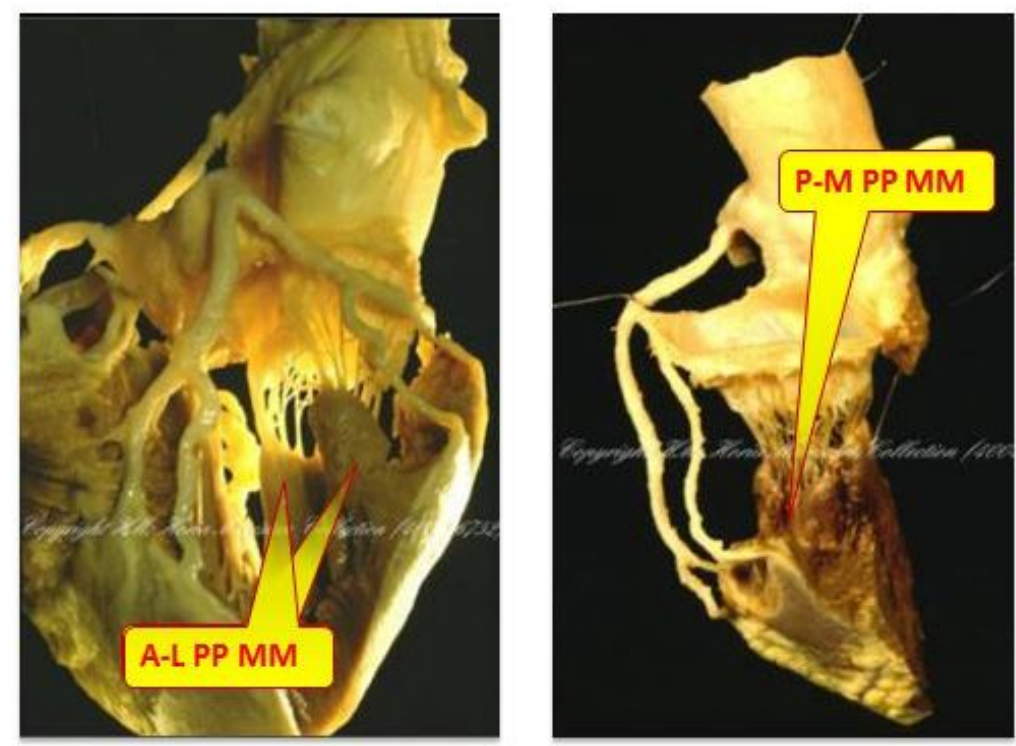

Figure 7. Differences in the vascularization of the papillary muscle. Postero-medial papillary muscle usually receives the blood only form the right coronary artery (rarely from circumflex artery), respect of the antero-lateral papillary muscle which has two sources of vascularization: left anterior descending artery and circumflex artery. By courtesy of MURESIAN Horia, MD, PhD - 2009. 
The acute ischemic mitral regurgitation (Fig 8) is more often due to structural lesions as complete or partial rupture of the papillary muscle, and less frequent due to an elongation of the muscle. The echocardiographic diagnosis may not be easy and it is often based on the use of the TEE in the emergency facility. The key issue in the successful management of these patients is timely intervention. If the clinical conditions and the logistic allow it, the patient should be urgently taken to the operating room. Most often, the acute ischemic regurgitation is surgically treated by mitral valve replacement. Mitral valve repair is rare, but, in specific cases, it may be a valid alternative. This depends on the quality and on the entity of the structural alteration of the subvalvular apparatus, and also on the experience of the surgical team.

From the echocardiographic point of view, the acute structural ischemic mitral regurgitation may present three different patterns: transection of papillary muscle ("too late" diagnosis), partial rupture of the papillary muscle ("timely" diagnosis) and elongation of the papillary muscle (the "tricky" echo diagnosis); see the scheme on the Fig 8.
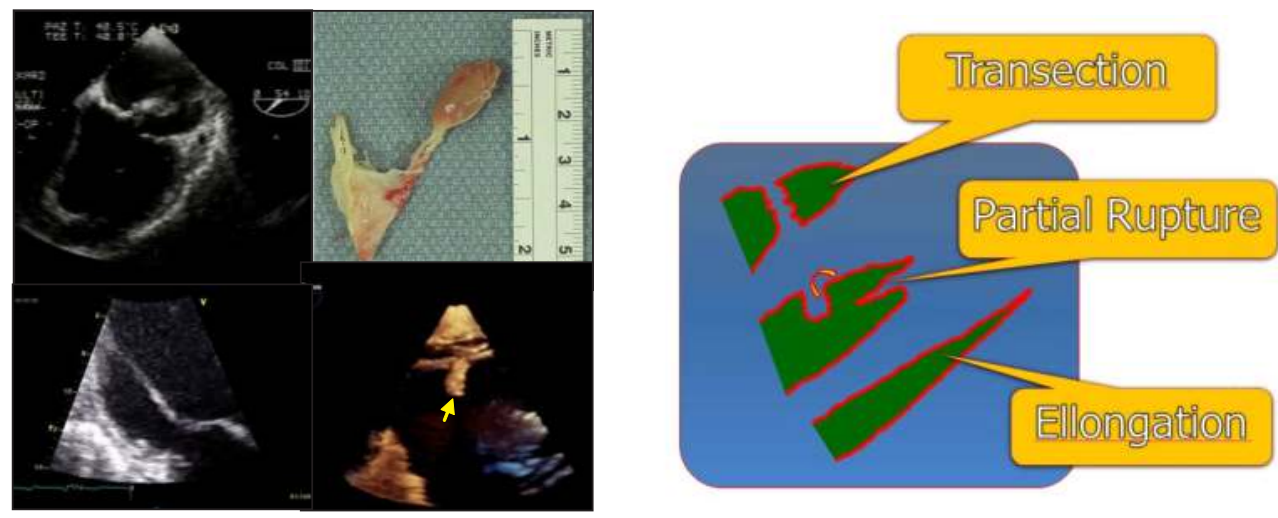

Figure 8. Echocardiographic classification of the structural acute ischemic mitral regurgitation (scheme). The upper pictures represent a case with transection of papillary muscle, in a patient with cardiogenic shock ("too late" echo diagnosis). In the bottom raw acute papillary muscle elongation (the first pictures on the left) and partial rupture of papillary muscle (right picture, yellow arrow); both patients were in $3^{\text {rd }}$ NYHA Class.

The gloomy clinical prognosis of acute significant ischemic mitral regurgitation associated to myocardial infarction (moderate or severe degree) is illustrated by its devastating impact on short- and long-term survival: almost one out of 4 patients die at one month and more than one out of 2 after one year (24\% early and 54\% 1-year mortality rate) (Otto, 2012).

In most cases the acute ischemic mitral regurgitation is a syndrome with conspicuous clinical implications. Generally, the clinical presentation of these patients is compatible with a hyper acute heart failure; they are highly symptomatic and may present with overt acute pulmonary edema, cardiogenic shock or even sudden death. Only minorities of patients are mildly symptomatic or asymptomatic and may present just a new systolic murmur.

Based on the echocardiography exam the acute ischemic mitral regurgitation may be divided in structural and functional. The structural ischemic mitral regurgitation is determined by 
complete papillary muscle rupture (transection), by partial detachment of one or both tips of a papillary muscle with subsequent elongation and by acute remodeling of papillary muscle with elongation (fig 8). In some cases the ischemic event may involve the point of insertion of the tendineous chord(s) which may result in a 'flail' which is an uncommon pattern for this pathology but may explain this kind of lesion.

In summary a myocardial infarction, in acute settings, may have one of the three structural damages on the papillary muscle: transection, partially rupture or elongation. The first two situations have already largely been described in many publications. This is not the case with the papillary muscle elongation, which is closer to chronic ischemic regurgitation and where the echocardiographic geometrical analysis, based on the triangle of coaptation, plays an essential role in diagnosis.

\subsection{Papillary muscle necrosis with complete rupture of papillary muscle}

Papillary muscle necrosis with complete rupture of papillary muscle is an uncommon but dramatic type of ischemic mitral insufficiency, with approx. $1.0 \%$ incidence in patients with myocardial infarction (Clements 1985). This is a life-threatening complication of myocardial infarction that requires a prompt diagnosis and surgical intervention (Fig 9). The posteromedial papillary muscle is more frequently involved because of its single coronary artery blood supply (Fig 7). Chordae to both leaflets arise from each papillary muscle so that, in cases of "complete" rupture, both leaflets are affected. The echocardiography exam generally shows loss of leaflets coaptation due to the floating ruptured papillary muscle ('flail' mitral valve). In these patients the echocardiographic diagnosis is relatively simple and, generally, may be done using transthoracic exam. These patients belong to the type $\mathrm{II}^{\circ}$ Carpentier's classification.

Nonetheless the complete rupture is rare; it carries a poor prognosis for the onset of acute pulmonary oedema and low output syndrome. Without surgery, the mortality is very high, up to $75 \%$ within the first 24 hours (Tcheng, 1992, Kirklin 2012). As a consequence, the echocardiographic diagnosis must rapidly be performed and the patient must be timely taken to the operating room. In most cases the intervention is a mitral valve replacement. However the operative mortality is high, ranging from 23 to $45 \%$, increasing in late referral. It may reach $57 \%$ in cases of reoperations (Loisance, 1990).

\subsection{Incomplete papillary muscle rupture}

Sometimes the papillary muscle is only partially ruptured. To avoid the high operative mortality in case of complete papillary muscle rupture, the diagnosis of papillary muscle rupture has to be done on this circumstance. Usually the clinical manifestation of this condition is an episode of acute heart failure occasionally with a relative stabilization and sometimes with a new systolic murmur. The echocardiographic diagnosis may be available using the transthoracic approach, but might also be relatively tricky and then the TEE should be preferred for the reasons mentioned above (unstable patient, unclear transthoracic view).

The echocardiographic presentation of this type of structural ischemic mitral regurgitation generally shows a prolapse of one leaflet; also the type $\mathrm{II}^{\circ}$ Carpentier's classification (which in 

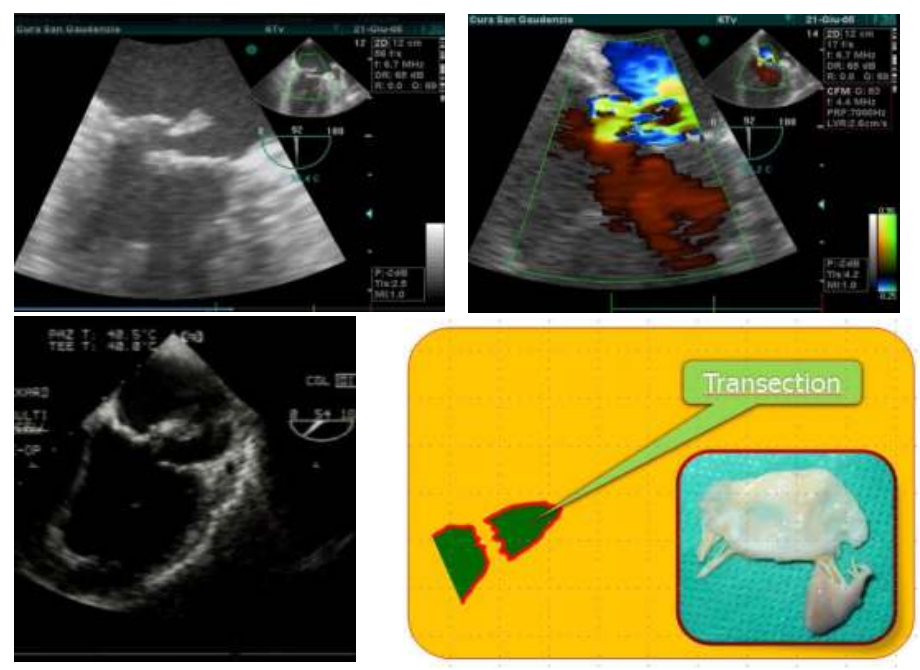

Figure 9. Acute severe structural ischemic mitral regurgitation due to transection of papillary muscle (2D TEE intraoperative exam). Note the loss of coaptation due to complete rupture of papillary muscle and flail (arrows). Both patients, with hemodynamic instability, were transferred to Cardiac Surgery Dpt. the same day of the transthoracic diagnosis. An emergency mitral valve replacement and CABG was successfully performed after coronary angiography.

reality is a 'false' mitral valve prolapse). An asymmetric apposition of the 'prolapsed' leaflet is also present. Often these patients are misdiagnosed as 'mitral prolapse', because the echocardiographer focuses the attention only on the mitral valve, neglecting to assess the papillary muscle. So, it is possible to fall into the trap, because at variance from the mitral valve, the papillary muscle is generally seen in a different echocardiographic plane, and often off axis view. If the echocardiographer doesn't have the concept of possible elongation of the papillary muscle due to the structural post-necrotic lesion, the true etiology of the lesion may be missed (Fig 10).

The take home message is to check explicitly the papillary muscle, searching it from different off axis views, starting from the clinical settings of the patient, essentially in case of abnormal geometry of the mitral valve. At any transthoracic suspicion of partial papillary muscle rupture the TEE exam must be done, chiefly in acute settings. The partial papillary muscle lesion may be otherwise missed and it may evolve to a complete rupture. At the echo, the ruptured head shows a hypermobile, redundant excursion which may mimic a degenerative lesion (e.g. Barlow disease). It is critical to remember that in most cases of papillary muscle involvement, massive mitral regurgitation is present and the surgery must be rapidly accomplished (Fig 11). In some cases mitral valve repair is possible depending on the intraoperative findings, the elapsed time from the acute myocardial infarction and the surgeon experience.

\subsection{Acute remodeling of the papillary muscle with elongation}

Acute remodeling of the papillary muscle with elongation is the third and rare form of acute ischemic mitral regurgitation which, usually, involves the inferior-posterior papillary muscle. 

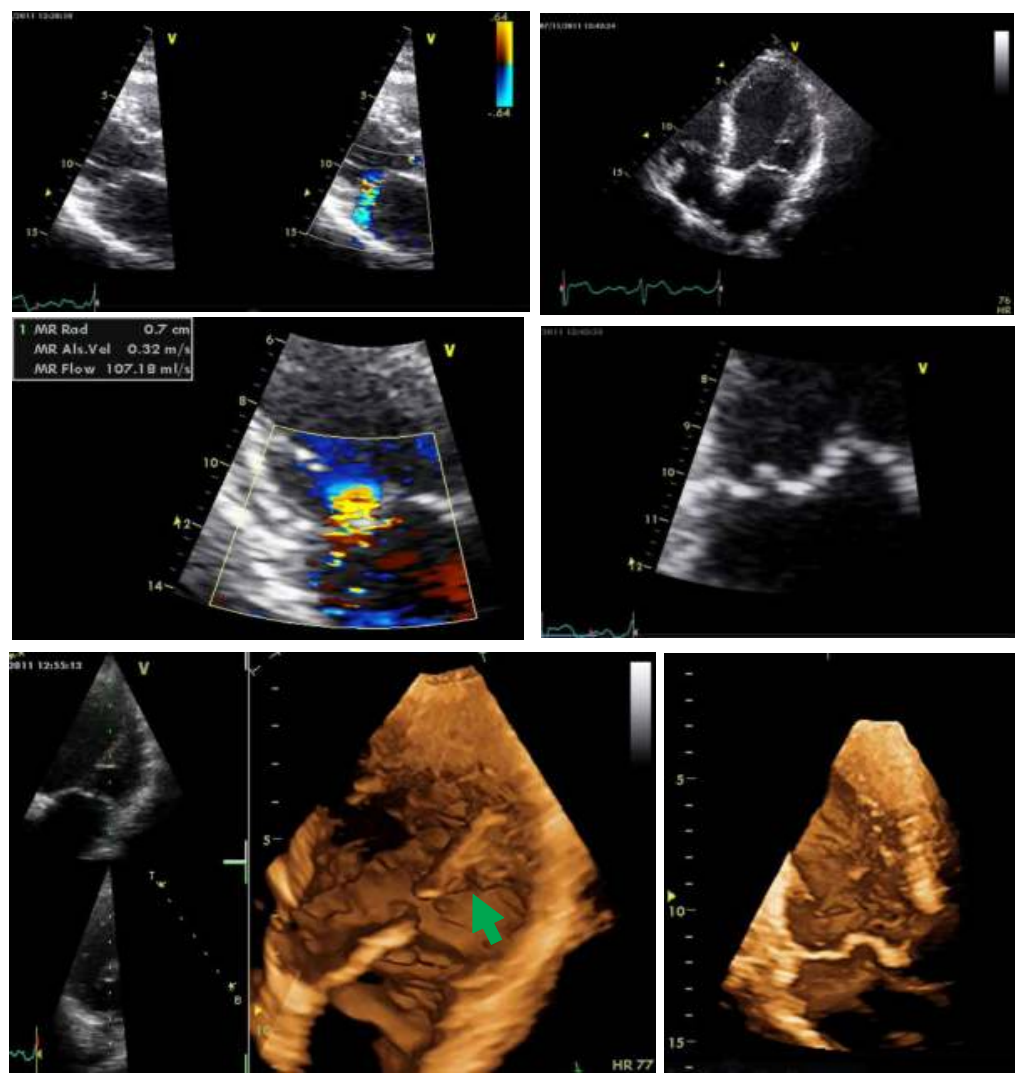

Figure 10. Ischemic mitral regurgitation due to acute elongation of papillary muscle, appearing as mitral valve prolapse. The pictures on the middle raw show prolapse of both mitral leaflet. In reality, only the focused examination on the papillary muscle shows the triangular shape of the muscle, with a sharp tip and a partial tearing beside (arrow, bottom raw, 3D TTE).

At the echo, the papillary muscle has a triangular shape and appears elongated and often slim (fig 12). This elongation determines a displacement of the coaptation point towards the mitral annular plane and to the left atrium, with subsequent mitral regurgitation. In this way the appearance of mitral valve is likewise similar with the mitral valve prolapse (type $\mathrm{II}^{\circ} \mathrm{Car}-$ pentier classification), but the etiology is the myocardial necrosis and not the anomaly of the connective tissue as in Barlow / Marfan disease.

The echocardiographic documentation of the ischemic etiology in these cases might be tricky. In order to better characterize the structural anatomy of the myocardium at the level of the infarcted or ischemic papillary muscle, additive methods could prove useful such as SPECT, cardiac MRI or PET, showing the myocardial scar or necrosis. Anyway, the diagnosis is extensively based on the geometrical analysis of the mitral valve apparatus performed by echocardiography. 

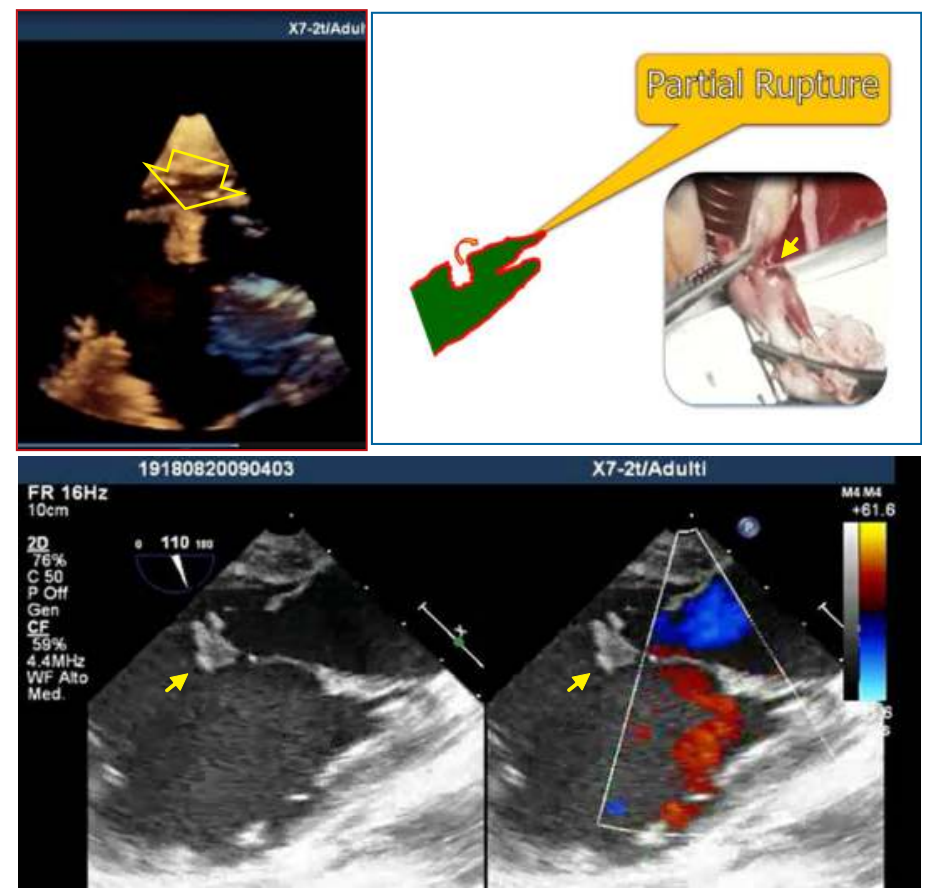

Figure 11. Partial rupture of papillary muscle (yellow arrows); TEE 3 D and 2 D exam and intraoperative picture.

The echocardiographic presentation of these patients shows a mitral valve with excess leaflets motion and prolapse, having the coaptation point situated 'very high' near the mitral annulus, due to an elongated papillary muscle. If the papillary muscle is difficult to visualize, the virtual tool of triangle of coaptation may be useful to suppose the true etiology, considering that the normal geometry of the mitral valve will be distorted, with disappearance of the standard triangle.
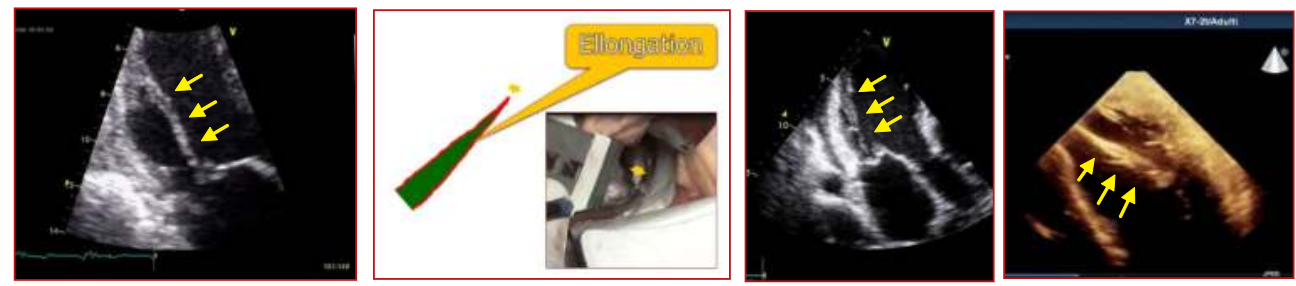

Figure 12. Acute post-ischemic elongation of papillary muscle (yellow arrows); scheme, intraoperative photo and 2D and $3 \mathrm{D}$ Echo. Note the tip of the papillary muscle (in the intraoperative picture and Echo slides) which reaches at the level of the mitral annulus. The papillary muscle is very thin, with a slim triangular shape and a very sharp tip: 'thorn papillary muscle'. In all cases the mitral valve presents severe regurgitation. Note the absence of the triangle of coaptation, due to the migration of the coaptation plane nearby the mitral valve annulus. 
The proper diagnosis and thus the management of such a serious clinical condition as the acute ischemic mitral regurgitation must rely on clear and robust echocardiographic concepts such as mitral valve geometry and functional anatomy. As already said the normal mitral valve geometry is the standard that guides both the evaluation of the various valve lesions and the surgical treatment. The central feature of the normal mitral valve geometry is represented by the triangle of coaptation (fig 4). The coaptation length (CL) and the coaptation height $(\mathrm{CH})$ are cornerstone elements used in order to fully describe the valve geometry and to assess the result of the repair (Cerin 2012). It may be assumed that from a three dimensional geometrical perspective the triangle of coaptation corresponds roughly to an asymmetrical tent. In the course of perioperative echocardiographic study, the systematical analysis of the mitral valve apparatus is done, focusing on whether the triangle of coaptation is present or not. This geometrical concept of the mitral valve allows the repair to be performed even in some of the serious cases of acute mitral regurgitation.

Nevertheless, it should be underlined that this condition, even if rare, is a very severe cardiological emergency, often characterized by low cardiac output and high early mortality; with medical therapy about $90 \%$ of patients with acute ischemic mitral regurgitation will died in the first two months. This is the reason why surgical correction must not be delayed. The predictors of better survival are: early operation, short duration of shock and mild degree of left/right ventricular impairment. For the management of these patients the use of intra-aortic balloon pumping and / or ventricular assist device (fig 13), out of inotropic pharmacologic support, is mandatory. Surgery might be delayed up to 2-3 weeks only when patient is stable; in this setting, as noted, the surgery is performed with lower risk.

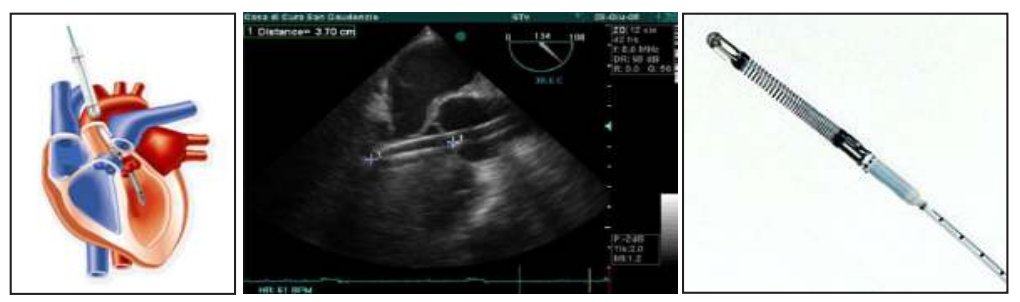

Figure 13. Acute heart failure due to ischemic mitral regurgitation. TEE intraoperative picture showing the assessment of the right position of the ventricular assist device (Impella) into the left ventricular outflow tract.

\section{Chronic ischemic mitral regurgitation}

\subsection{Physiopathological consideration}

Largely, the chronic ischemic mitral regurgitation is considered a long-lasting developing process of LV remodeling after myocardial infarction, causing mitral regurgitation by dysfunction and distortion of the components of the mitral valve including the chordae, the annulus and the leaflets. Nevertheless, this is not always true: some patient with acute 
infarction may develop an acute and dynamic process of ventricular remodeling involving the papillary muscle, which may not be perceived or checked in real time at echo and discovered later, as 'chronic' mitral regurgitation.

From this point of view, the classification of ischemic mitral regurgitation in acute and chronic may be in some cases misleading; ischemic mitral regurgitation is a ventricular disease, following the same steps as the myocardial infarction. So, as the myocardial infarction recognizes an acute period, a recent (sub-acute) and then a stable (chronic) phase, it seems logical to use the same classification for ischemic mitral regurgitation. In the era of shorter hospitalization of infarcted patients the acute ischemic mitral regurgitation, being a dynamic phenomenon, may be missed at the first echo examinations and 'discovered' over time, as 'late' complication (Popa BA 2007) (fig 14); Thus, the echocardiographer has to be aware that the classification in acute or chronic may reflect mainly the moment of diagnosis and less the true period of time when the lesion occurred. This aspect is important as regard the prognosis of ischemic mitral regurgitation, because, as noted, the acute and sub-acute ischemic mitral regurgitation have a worse prognosis over a short period of time, respect of chronic ischemic mitral regurgitation.
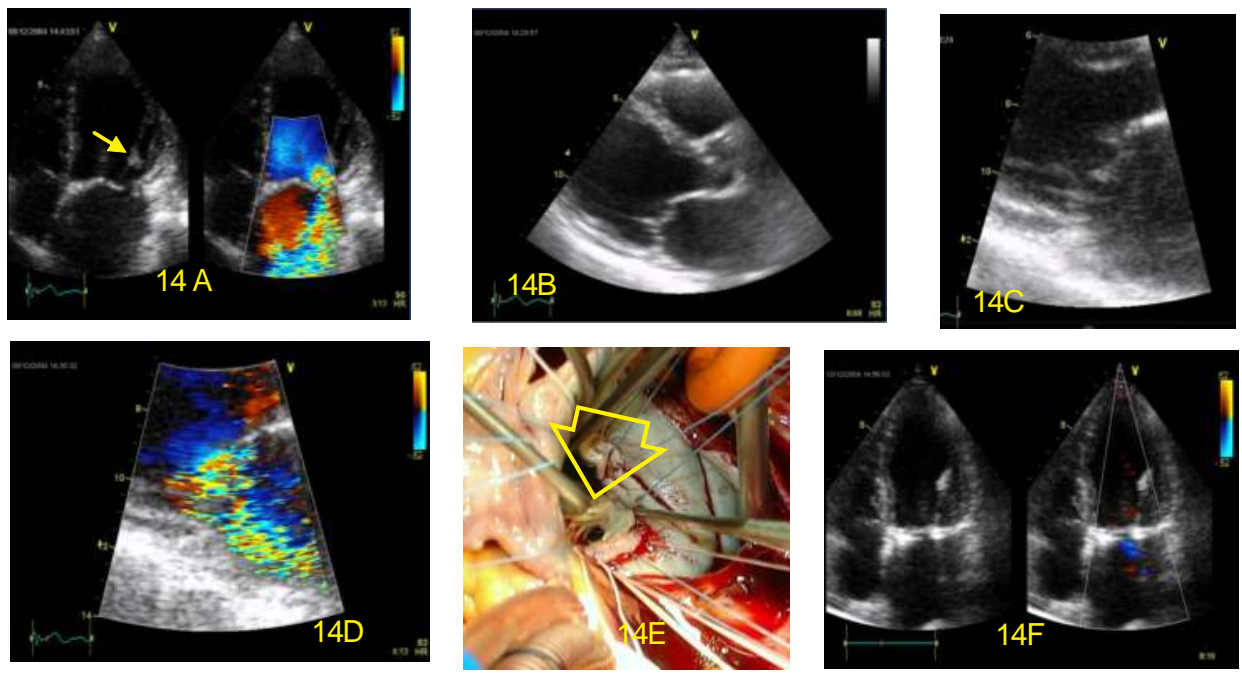

Figure 14. Severe ischemic mitral regurgitation (14D) diagnosed by TTE 10 years after an acute 'uncomplicated' myocardial infarction. The clinical presentation of the patient was exertion dyspnea. Ten years before, at discharge from the hospital the Echo exam has been normal. Note the elongation of the papillary muscle (14 A,C,E), causing loss of leaflet coaptation (14 $A, C$ ), with marginal prolapse of $A_{2}$ scallop (14B,C). Apparently the patient presents an $A_{2}$ localized prolapse (14BC), which, in reality is caused by an elongated papillary muscle. The tip of the papillary muscle is shown (14E, yellow arrows), arriving till the mitral annular plane (which is categorically abnormal). The transthoracic postoperative echo after mitral repair (mitral ring + suture and shortening of the papillary muscle), shows trivial residual mitral regurgitation (14F). 
The chronic ischemic mitral regurgitation, comparing with the acute one, is a much more gradual and more complex process, because of the slow progression over time of the left heart remodeling (fig 14). This clinical condition recognizes two main mechanisms: a structural alteration of the subvalvular apparatus (papillary muscle and the corresponding LV wall), alongside of the phenomenon of left ventricular cavity remodeling.

\subsection{Left ventricle post infarction remodeling process}

From the physiopathological point of view the left ventricular post myocardial infarction remodeling is considered to be a phenomenon which can be divided in two parts: a regional and a global process of remodeling.

Regional remodeling is more often found after the inferior infarction (but not only), and may evolve over time in two different ways:

1. mainly a process of inferior wall remodeling with displacement of the papillary muscles. This type is associated with overt systolic restriction of posterior mitral leaflet.

2. inferior wall remodeling and displacement of the papillary muscles, combined with postischemic elongation of papillary muscles. In this kind of inferior post infarction remodeling the systolic restriction of the posterior mitral leaflet may be less evident or even absent in some cases. Respect of the first type, the geometry of mitral valve in this sort of ventricular remodeling will be different.

Global left ventricular remodeling is generally seen after two heart attacks (multiple myocardial infarctions and in cases with ischemic dilated cardiomyopathy) and it is characterized by restriction of both mitral leaflets, tethering of the mitral valve apparatus with mitral valve tenting, due to progressive migration of the papillary muscles simultaneously with overall left cavity dilatation.

In the patients with left ventricular remodeling and chronic ischemic mitral regurgitation, these physiopathological concepts are expressed by two main echocardiographic patterns of left heart remodeling:

a. the echocardiographic pattern of limited LV remodeling: inferior or lateral infarction scar, mild left ventricular dilatation, mild systolic dysfunction and chronic ischemic mitral regurgitation due to unambiguous systolic restriction of posterior leaflet (fig 15), and

b. the echocardiographic pattern of extensive LV remodeling: major left ventricular cavity dilatation with dysfunction and distortion (by anterior and inferior myocardial infarction), typically with poor systolic function, but also with a diastolic one and the tethering of both mitral leaflets (fig 3).

Both types of chronic ischemic mitral regurgitation present a mitral annular dilatation, obviously more evident in patients with double myocardial infarction. A form of symmetric remodeling of the mitral valve apparatus is present in patients with ischemic dilated cardiomyopathies ('extensive' LV remodeling) due to bilateral migration of both the papillary muscle (scallops $\mathrm{P}_{1} /$ $\mathrm{P}_{2} / \mathrm{P}_{3}$ ). Conversely, a limitary process of mitral valve apparatus remodeling is present in patients 

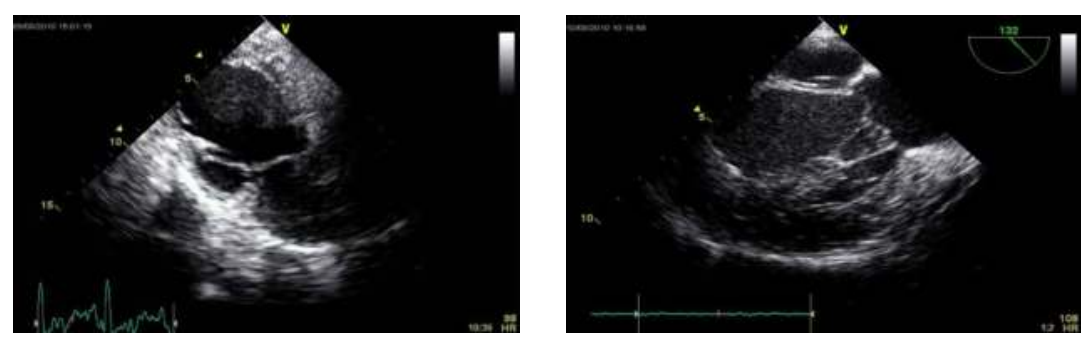

Figure 15. Limited LV remodeling: TTE (left) and TEE 2-chamber view (right) showing systolic restriction of the posterior mitral leaflet and minimal leaflet coaptation. Necrosis of the inferior wall is evident, with mild left ventricular dilatation (EDVol 170ml), mild systolic dysfunction (EF 43\%) and chronic ischemic mitral regurgitation due to clear systolic restriction of posterior leaflet.

with 'limited' LV remodeling (inferior infarction) and asymmetric mitral valve remodeling, due to solitary migration of the postero-medial papillary muscle (scallops $\mathrm{P}_{3} / \mathrm{P}_{2}$ ).

These two main echocardiographic patterns of LV remodeling, when associated with chronic ischemic mitral regurgitation, are easy to identify by transthoracic echocardiographic exam and the transesophageal examination is not necessary. Sometimes, these configurations may be less definite, as in patients with regional or global remodeling, combined with coincident papillary muscle elongation. In these cases there is an additional phenomenon of migration of the LV infarcted wall, associated with post ischemic elongation of the papillary muscle.

A geometrical analysis of the mitral apparatus, by use of the echocardiographic tool of triangle of coaptation may identify these patients with regional or global remodeling, combined with elongation of the papillary muscle. As already has been mentioned, the patients with papillary muscle elongation present a distortion of the triangle of coaptation with progressive alteration of the standard trilateral shape (fig 5), till the complete disappearance of it (fig.6). The mechanism of mitral insufficiency in these patients is more complex and the surgical strategy has to be tailored accordingly.

The elongation of the papillary muscle may often be unnoticed if the operator mostly focuses the attention on the mitral leaflet, on the valve coaptation and on the degree of mitral regurgitation. Moreover, the papillary muscles are difficult and rarely seen in the same plane with the mitral valve leaflets, being sited distant from the coaptation valve plane; only a specific and vigilant off axis assessment of them may reveal the muscularly post-ischemic remodeling.

When the papillary muscles elongation is obvious, the surgical strategy has to be adapted and sometimes a shortening of them or use of the artificial chordae may be necessary to restore a good plane of valve coaptation and valve geometry.

Therefore, as message for the everyday practice, in acute or in chronic ischemic mitral regurgitation, the marker of the papillary muscle elongation is the distorted coaptation triangle.

Consequently, in the analysis of ischemic mitral regurgitation the starting points are restricted motion of the posterior mitral leaflet (type $\mathrm{III}^{\circ}$ Carpentier's classification), combined with mitral annular dilatation, secondary to migration of the wall and left ventricular enlargement 
(fig 15). The consequent mitral regurgitation leads to left ventricular volume overload, closing the vicious circle of ischemic mitral regurgitation and of left ventricular remodeling. The echocardiographic examination will show the tethering of one or both mitral leaflets, which is the 'standard' pattern of chronic ischemic mitral regurgitation. However, around $10 \%$ of patients with chronic ischemic mitral regurgitation may show a different pattern of regurgitation with 'false mitral prolapse', by elongation of the papillary muscle due to local ischemia, necrosis and/or fibrosis (false because the etiology is not degenerative, but ischemic one).

In these patients, from the echocardiographic point of view, there are three elements that may suggest the suspicion of ischemic etiology of the prolapsed valve:

1. leaflets - prolapse limited to one of the mitral leaflets, often of the anterior one,

2. papillary muscle - specific appearance of the papillary muscle: elongated, hyperechogenic, thinning and triangularly shaped; "thorny" papillary muscle (fig 16);

3. valve geometry - the abnormal mitral valve geometry, with distortion of the triangle of coaptation, until its complete extinction (see also the Fig 5, 6)
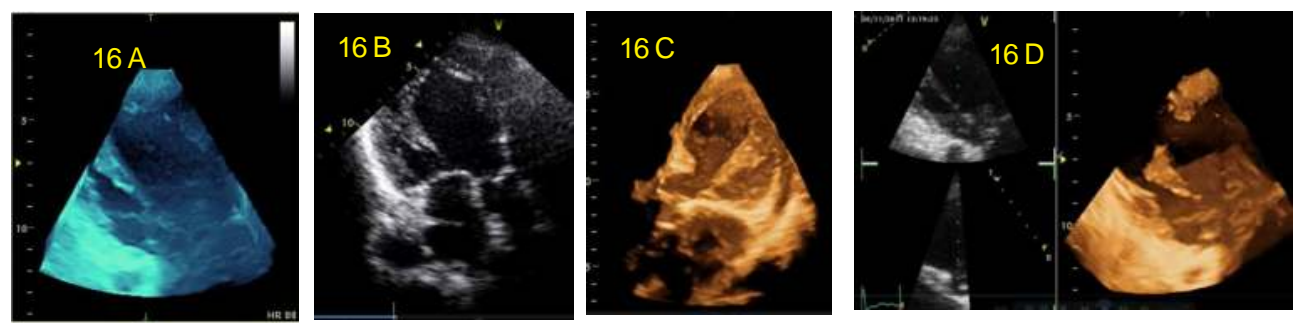

Figure 16. $A, B, C, D$. Four different cases with chronic post ischemic elongation of the papillary muscle: $2 D$ transthoracic exam (16B) and live 3D (A,C,D). Note the progressive thinning of the papillary muscle, with the sharp aspect of the tip of it: so called 'thorny papillary muscle'.

Chronic ischemic mitral regurgitation may also be synonymous with ruptured tendineous chordae. Due to ischemia or necrosis the insertion place of the mitral chords on the tip of the papillary muscle may become friable, and often a limited 'ischemicflail', may be apparent (fig 17).
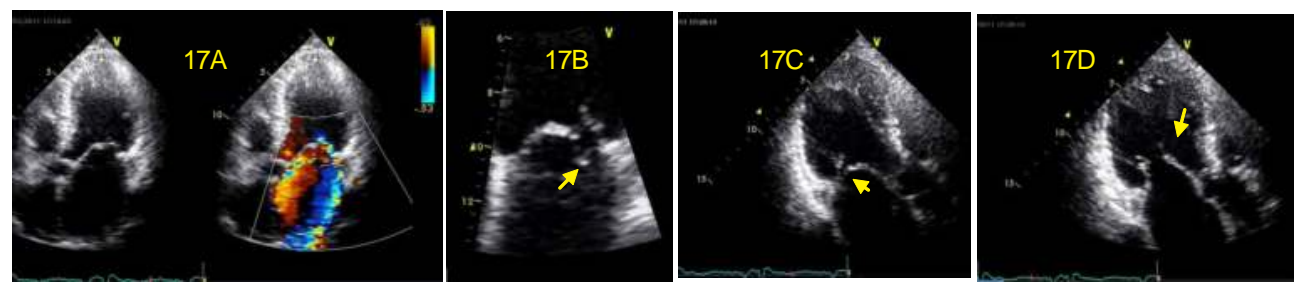

Figure 17. Loss of leaflet coaptation (B,C), with flail (B) and eccentric jet. The free ruptured chordae is visible in systole (C) and also in diastole (D). The pictures belong to the same patient. 
Indication to surgery: With an ever increasing population of patients with coronary artery disease complicated by chronic ischemic mitral regurgitation, the question whether to perform a mitral valve repair in addition to CABG is one of the most common and controversial clinical dilemmas faced by cardiac surgeons and cardiologists today. There is a general agreement regarding the indication for surgery in patients with severe ischemic mitral regurgitation. The problem arises for moderate ischemic mitral regurgitation. At present, there is no definitive randomized, prospective trial that clarifies which approach should be taken. The 2011 ACCF/ AHA guidelines on CABG state that patients undergoing CABG who have severe ischemic mitral valve regurgitation not likely to resolve with revascularization, should have concomitant mitral valve repair or replacement at the time of CABG (Class I, Level of evidence B) (Gillinov 2001, Trichon BH 2003, Fattouch K 2009 \& 2010, Zoghbi W 2003). Operative mortality is considered to be around 10\% (Rankin 1988, Cohn 1995, Grossi 2001). Some studies advocate mitral valve repair to all patients that undergo CABG even when the degree of mitral regurgitation is mild. This strategy seems to be reasonable if the chronic ischemic mitral regurgitation is considered to be expression of the LV deformation / remodeling process, which is not still reversible in the chronic phase of the myocardial infarction. A careful echocardiographic analysis focused mainly on the quality of leaflet coaptation and on the extension of LV deformation (limited or extensive remodeling), may guide the surgeon.

\section{Assessment of ischemic mitral regurgitation severity}

Due to the hemodynamic conditions in ischemic heart disease and the particular shape of the regurgitant orifice, assessment of the severity of mitral regurgitation may be challenging. Mitral regurgitation in an ischemic setting has a profound impact on patient's prognosis and therefore its quantification plays a key role in the revascularization plan because moderate and severe mitral regurgitation have indication to surgery. Given this special impact on prognosis, the thresholds of defining a severe mitral regurgitation in ischemic patients are much lower than in other contexts (see below the PISA method).

The qualitative method, based on regurgitant jet area, is not reliable. This is true especially when an exact assessment of mitral regurgitation is need, because this approach is prone to many biases, being so dependent upon loading conditions and on equipment settings (for example color gain). The gradient between left ventricle and left atrium will highly influence the size of the jet. In an acute mitral regurgitation, in which left atrium pressure is elevated and the left ventricle-left atrium gradient is low, the regurgitant jet may be small, even though the real regurgitant volume is important. Conversely, in a high blood pressure setting (i.e. a high left ventricle-left atrium gradient) the regurgitant jet will be bigger. Last but not the least, eccentric jets (like the ones found in posterior leaflet restriction), seem smaller than the central ones due to Coandă effect (hugging of the adjacent wall of the left atrium).

The semi-quantitative (vena contracta) and quantitative methods (PISA) are therefore advisable whenever their determination is possible. 
Vena contracta is the narrowest region of the regurgitant jet, which is found not at the level of the leaflets, but just below the regurgitant orifice (because the blood is still converging for a few millimeters before re-expanding in left atrium) (fig 18). Its width is a marker of the regurgitant orifice area. It should be measured in a window perpendicular to the coaptation line, like parasternal long axis or four chambers views. A value of $<3 \mathrm{~mm}$ is compatible with a mild regurgitation, while a value of $>7 \mathrm{~mm}$ is indicative of severe regurgitation. Values inbetween are not diagnostic of a moderate mitral regurgitation, because of an important overlap. In ischemic mitral regurgitation, the shape of the regurgitant orifice is not round, but rather elliptic. For this reason, assessment of vena contracta width should be done in two orthogonal planes (e.g. parasternal and apical four chambers) and an average of these two should be reported. A value $>8 \mathrm{~mm}$ is diagnostic of severe regurgitation. The $3 \mathrm{D}$ echo can be a solution for assessment of vena contracta in an ischemic setting.

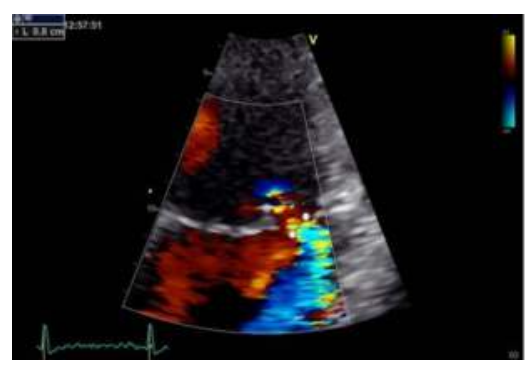

Figure 18. Vena contracta depth measurement at the narrowest point of the regurgitant jet. The three components of the jet are visible: convergence zone, vena contracta and the flow into the left atrium.

Quantitative approaches and especially PISA (proximal isovelocity surface area) are the most indicated. PISA is based on the fact that near the regurgitant orifice, the blood (which should pass throw a restricted area) is disposed in hemispheric layers, having the same velocity at a certain distance from the orifice. Lowering the Nyquist limit at $15-40 \mathrm{~cm} / \mathrm{s}$ (the velocity at which aliasing is appearing), all the "layers" with a higher velocity will have the aliasing phenomenon. Measuring the first aliasing hemisphere we'll have a marker of regurgitation degree.

PISA method permits assessment of:

- the flow rate (FR) as $F R=2 \times \pi \times R^{2} \times \mathrm{Val}$

where $R$ is the PISA radius and $\mathrm{Val}$ is the aliasing velocity

- effective regurgitant orifice area (ROA) as $E R O A=F R / V \max$

where $F R$ is the flow rate and $V$ max is the maximum velocity measured on mitral regurgitation envelope in continuous Doppler

- regurgitant volume $(\mathrm{RV})$ as $R V=R O A \times T V I$, where $E R O A$ is effective regurgitant orifice area and TVI is the time-velocity integral measured on mitral regurgitation envelope in continuous Doppler (fig 19). 

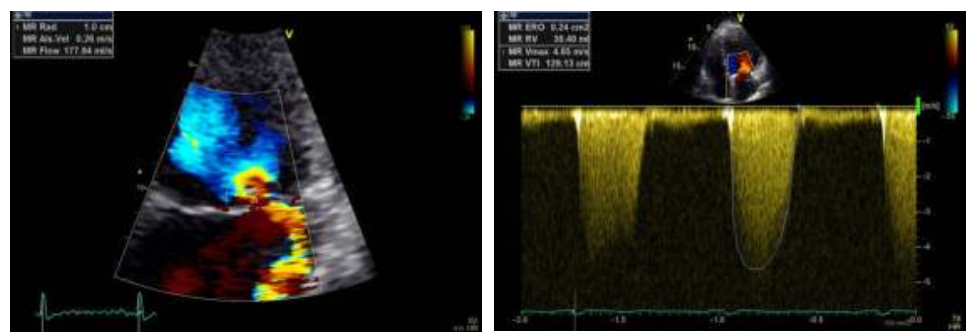

Figure 19. Assessment of mitral regurgitation severity according to PISA method (MR ERO, MR Flow). The baseline of color Doppler is moved toward the regurgitant jet direction and the hemisphere radius is measured (left). On the mitral regurgitation envelope (continuous Doppler) maximum velocity and TVI are determined

In eccentric jets (posterior leaflet restriction) the largest PISA is to be found in the parasternal long axis view, while in central jets the apical four chambers view should be used.

The elliptic shape of regurgitant orifice found in ischemic mitral regurgitation leads the PISA method to underestimate the real regurgitant volume (PISA is bigger than a hemisphere). Considering the profound deleterious effect of mitral regurgitation in an ischemic context, cut off levels of severity are lower than in organic mitral regurgitation, i.e. an EROA more than $20 \mathrm{~mm}^{2}$ (vs. $>40 \mathrm{~mm}^{2}$ in organic mitral regurgitation) and a regurgitant volume greater than $30 \mathrm{ml}$ (vs. $>60 \mathrm{ml}$ ) defines a severe ischemic mitral regurgitation.

Due to the dynamic characteristic of ischemic mitral regurgitation, PISA is not the same throughout the whole systole, being usually smaller in mesosystole when the regurgitation tends to decrease.

If hemispheric floor is not flat (i.e. $180^{\circ}$ ), an angle correction can be made. In this situation

$E R O A$ adjusted $=E R O A \times \alpha / 180$, where $\alpha$ is the angle of convergence.

In multiple jets, both vena contracta width and PISA method are not additives and cannot be used.

Surgical approaches. Overview. The surgical treatment of acute or chronic ischemic mitral regurgitation is strongly influenced by the echocardiographic assessment, which remains the only tool for surgical planning and postoperative control. The complex mechanisms of ischemic mitral regurgitation are analyzed by echo and the surgical planning is done after transthoracic and usually elaborate after intraoperative transesophageal examination. Based on echocardiographic analysis, various approaches have been proposed for the management of ischemic mitral regurgitation. So far, both medical treatments and classical surgical techniques are not always sufficient. Within the next paragraphs a rapid overview of some of the various surgical techniques implied in the treatment of ischemic mitral regurgitation will be done.

1. Undersized mitral valve annuloplasty. The strategy of over-reduction of mitral annulus, combined with the coronary by-pass surgery is the most diffused surgical approach, especially in patients with dilated heart and chronic ischemic mitral regurgitation. As 
noted, this technique addressed just the annular dilation. The echocardiographic examination plays an essential role because it confirms the dilation of the mitral annulus, and the subsequent reduced and/or loss of leaflet coaptation in case of advanced left ventricular and annular dilatation ('extended' LV remodeling pattern). As a rule, Echo indicates the presence of tethering of the mitral leaflets, and also the systolic and, in cases with advanced heart remodeling and ischemic dilative cardiomyopathy, the diastolic restriction of the posterior mitral leaflets.

Concomitant coronary revascularization and downsizing of the mitral annulus by prosthetic rings, showed promising results with $80 \%$ good competence of the mitral valve at 2-years follow-up, but only 50\% at five years (McGee, 2004, Klein 2011). Better results were published by Dion (2004) according to a proper algorithm. Patients treated with this technique showed almost always the echocardiographic picture of the so-called "monoleaflet" mitral valve, where the anterior leaflet covers the entire mitral orifice and the posterior leaflet remains restricted in its diastolic position. At the time of surgery the preoperative TEE Echo assists the surgical mitral repair by estimating the length of the medial scallop of the anterior mitral leaflet (scallop $\mathrm{A}_{2}$ ). This is usually performed from the distal-esophageal $135^{\circ}$ view (three chamber view) and is essential in choosing the size of the annuloplasty ring. The choice of the size of the prosthetic ring is still empirical, but usually a ring two to three sizes smaller than measured is used.

The other determinant of the ischemic mitral regurgitation in the advanced stage of left ventricular remodeling is the restricted motion of the posterior mitral leaflet and loss of the anatomical and functional leaflet reserve both in systole and also in diastole. The existence of the anatomical and functional reserve of posterior mitral leaflet is a key element for having a competent mitral valve and, unfortunately, this aspect is not resolved by the over-reduction technique alone. To overcome the leaflet restriction some authors cut a limited number of basal chordae tendinae with the intent of reducing leaflet tethering and improving the coaptation. This simple procedure reduced tenting without creating valve prolapse, and the consequence is a decrease in mitral regurgitation (Messas 2001).

2. Because of these unsatisfactory results with the undersizing technique alone, the repositioning of the posterior papillary muscle has been proposed, mainly in patients with ischemic dilated cardiomyopathy, as adjunctive approach to the annuloplasty; the outcome is currently under evaluation (Hvass 2010). The role of intraoperative TEE is to define the deformation of the subvalvular apparatus from the geometrical point of view. On one hand, it must preoperatively assess the distance between the base of the papillary muscles (usually from the transgastric short axis view), and on the other hand it has to evaluate the geometry of the left ventricle using the sphericity index.

3. Elongation of the posterior mitral leaflet with pericardial patch (LEX), represents a relatively novel surgical approach to relief ischemic systolic restriction, despite some controversy regarding this technique (fig 20). Some encouraging results of LEX were reported but the number of treated patients is, so far, very limited (Dobre 2000, Langer 2006, de Varennes 2009). Surgical leaflet extension by autologous pericardial patch addresses directly the problem of ischemic leaflet restriction and tethering (type $\mathrm{III}^{\circ} \mathrm{b}$ 
Carpentier classification). It is a physiologically coherent and relatively simple approach, intended to increase the surface and the mobility of the posterior mitral leaflet, in order to allow a better leaflet coaptation.
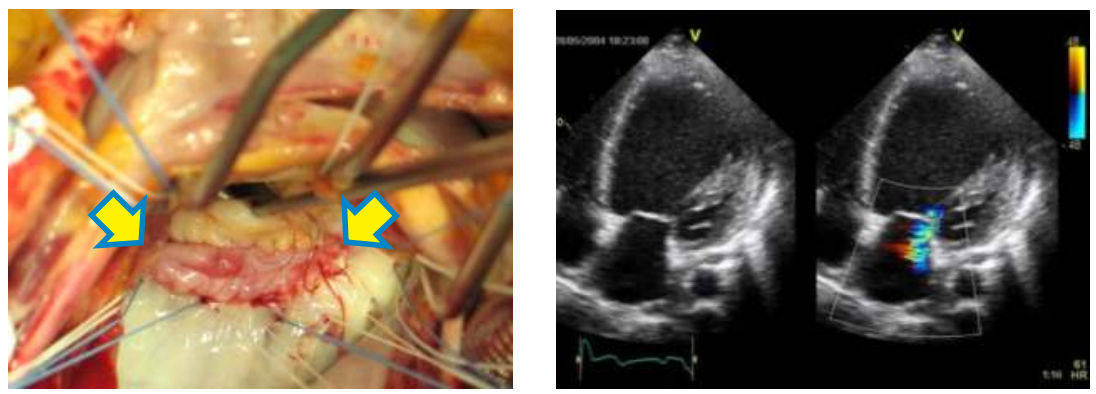

Figure 20. Posterior mitral leaflet after extension with pericardial patch (arrows) in a patient with ischemic dilated cardiomyopathy: extended LV remodeling (intraoperative view - left and TTE preoperative picture; right).

This technique allows the extension of the surface of the posterior leaflet, especially in the area of the scallops $\mathrm{P}_{3}$ and $\mathrm{P}_{2}$, which is normally more restricted by the post-ischemic tethering. Furthermore, this procedure provides an additional anatomical leaflet reserve: in case of continuing left ventricular remodeling after surgery, which may be present in about $50 \%$ of the patients, according to some authors. Leaflet extension by autologous pericardial patch has been widely and successfully used in the reconstruction of other mitral valve pathologies such as rheumatic disease and endocarditis, with acceptable long-term results (Zegdi, 2007). This procedure can be performed within 15-20 extra minutes of aortic cross-clamping, and allows implanting larger sizes mitral prosthetic rings, with minor diastolic gradient through the mitral valve. Further studies with larger number of patients and adequate long-term follow-up are needed to assess the efficiency of this simple and promising approach to the treatment of ischemic mitral regurgitation, in case of ischemic dilated cardiomyopathy.

Once more the echo plays an essential role by identifying the patient candidates to LEX. Typically the Echo aspect demonstrates small posterior mitral leaflets, systolic and, sometimes, diastolic leaflets restriction, with significant mitral regurgitation due to reduced coaptation and asymmetrical apposition of the anterior leaflet. Consequently, at the postoperative examination Echo evaluates not only the mitral valve function as expressed by the degree of residual regurgitation, but, more important, the anatomical (leaflet length) and functional reserve (the coaptation length).

For every day practice three main situations will be considered:

1. Chronic ischemic mitral regurgitation determined by restriction of the posterior leaflet and apical displacement of the papillary muscle in patients with inferior myocardial infarction (or inferior and lateral myocardial infarction), mild left ventricular dilatation and mild systolic dysfunction: so called 'limited' LV remodeling pattern. 
2. Chronic ischemic mitral regurgitation by systolic (and sometimes diastolic) restriction of mitral leaflets motion (tethering of mitral leaflets) in patients with history of double or multiple infarction, overt left ventricular dilatation and depression of systolic function (ischemic cardiomyopathy): 'extended' LV remodeling pattern.

3. Chronic ischemic mitral regurgitation by papillary muscle elongation (with various degree of false prolapse of the mitral leaflets) (Hashim 2012); usually patients with inferior myocardial infarction, chronic ischemic mitral regurgitation and distortion of the coaptation triangle.

\subsection{Chronic ischemic mitral regurgitation due to restriction}

Chronic ischemic mitral regurgitation due to restriction of the posterior mitral leaflet and asymmetrical apposition of the anterior one, by apical displacement of the posterior papillary muscle and left ventricular wall. Fig 21.
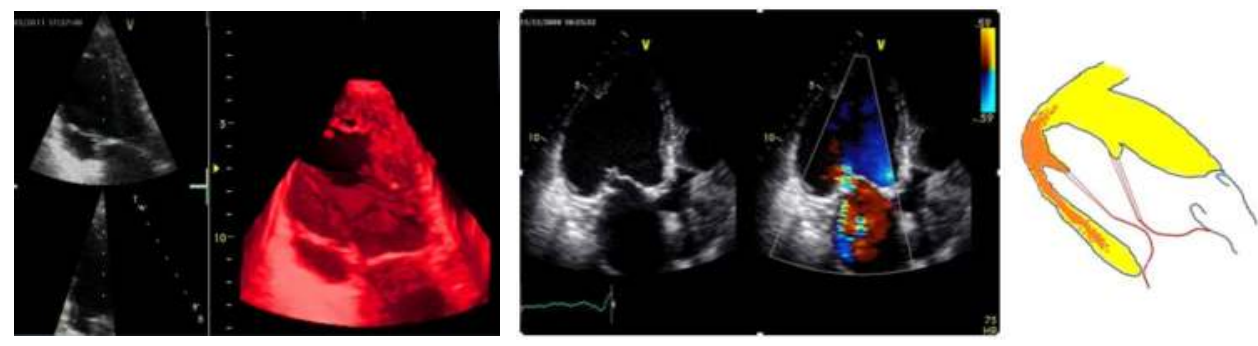

Figure 21. Systolic restriction of posterior papillary muscle; 3D and 2D transthoracic echocardiography and drawing.

Background. This is the most common type of ischemic mitral regurgitation (about $2 / 3$ of all cases of chronic ischemic mitral regurgitation), belongs to the Carpantier's type III $^{\circ} \mathrm{b}$ classification and has been extensively described. The valve leakage is here the result of alteration of subvalvular apparatus secondary to left ventricle remodeling following myocardial infarction. Frequently, they present with a myocardial scar caused by a previous myocardial infarction and the remodeling process of the left heart is less expressed, with mild systolic dysfunction. Respect of patients with ischemic dilated cardiomyopathy, this category of patients has less dilatation of the left ventricular cavity, and, as a rule, history of inferior (or lateral) myocardial infarction: echocardiographic pattern of limited LV remodeling. An infarction at the basal side of the left ventricle, involving the postero-medial papillary muscle will lead to a posterior and apical displacement of the papillary muscle, which will pull the posterior leaflet toward the posterior wall, impeding it to arrive to the normal coaptation plane during systole (figure 22). The postero-medial papillary muscle is more prone to ischemic remodeling than the antero-lateral one, having only a vascular source (from circumflex or right coronary), while the latter has a double vascularization (from both left anterior descending and circumflex) see also the fig 7 . 
Geometrical echocardiographic analysis. As already mentioned, the echocardiographic presentation of systolic restriction of the posterior mitral leaflet is typical: in presence of an inferior and/or lateral myocardial infarction, the posterior leaflet appears fixed in systole (and sometimes also in diastole), instead of the normal thickness of it and of the absence of fibrosis and/or calcification. The anterior leaflet is overlapping the posterior restricted leaflet (asymmetrical apposition), due to the deficit of a supporting surface in systole. The transthoracic exam is sufficient to identify the mechanism of mitral regurgitation and, equally important, to evaluate the degree of mitral regurgitation (Monin JL, 2005). The algorithm of evaluation of mitral valve geometry should be focused on the assessment of the triangle of coaptation and coaptation length (normally $>6 \mathrm{~mm}$ ). In this specific condition there is an insufficient leaflet coaptation due to systolic restriction of the posterior leaflet, asymmetric apposition of the anterior leaflet and annular dilatation.

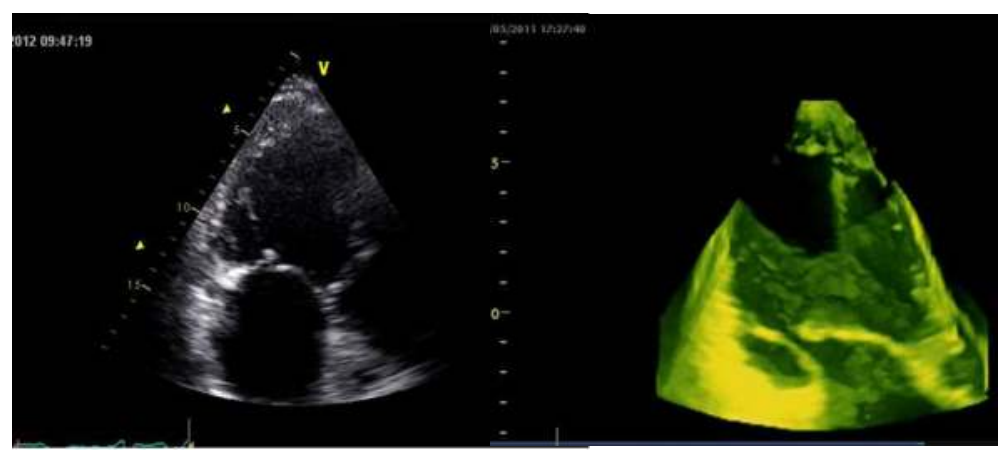

Figure 22. Restriction of posterior leaflet with typical asymmetric apposition of the anterior one (2DE and 3DE TT pictures) in a patient with inferior MI. The tip of the anterior mitral leaflet is heading towards the left atrium due to chronic loss of the systolic resistance plane.

The evaluation of the anatomical and functional reserve of the posterior mitral leaflet plays an essential role for those patients who will undergo reconstructive surgery. For example, when the posterior leaflet is too small ( $<1 \mathrm{~mm}$ of length) and particularly restricted (fixed leaflet), it is a clear sign of reduced anatomical reserve. This key point will indicate the need of a more aggressive surgical approach where reductive annuloplasty should be associated to leaflet extension as discussed before.

Echo-surgical messages: As general rule, in coronary patients undergoing revascularization, the assessment of the severity of valve regurgitation (and so of decision to treat the regurgitation), should be done by transthoracic exam, as general anesthesia may significantly decrease the severity of regurgitation. When necessary, in the operating room, a preload and/or afterload test provides an additional estimation of the severity of mitral regurgitation (Aklog 2001, Gisbert A, 2006). Intraoperative transesophageal echocardiography is routinely performed in order to confirm the mechanism of mitral regurgitation and mainly to control the outcome of repair. 
Surgical planning and management. Most of the times, the surgical technique consists in mitral annuloplasty using an undersized complete prosthetic ring or in alternative only a posterior prosthetic ring which should reduce the annular dilation especially in the region of the medial scallops $\left(\mathrm{P}_{3}\right)$. Depending on the functional and anatomical reserve of the posterior leaflet, cusp extension using a pericardial patch may be taken into consideration. The newly inserted pericardial patch should be as large as possible because, by sewing usually it remains smaller and also because it has a natural tendency to shrink overtime.

\subsection{Ischemic dilatative cardiomyopathy (fig 23)}

Background. This situation is the final stage of an unfavorable evolution of ischemic heart disease. Usually, in anamnesis, there are more than one myocardial infarction and a long story of percutaneous interventions and/or surgical treatments.

Mechanism. This category of patients presents the phenomenon of "mitral - ventricular mismatch". Due to progressive dilatation of the left cavity, the rightly balance between the mitral valve apparatus and the dimension of the multiinfarcted left ventricle will be lost, and in this condition, the mitral valve will become insufficient. Any tentative to repair the mitral valve has to take into consideration this aspect. More remodeled and dilated the left cavity will be, more difficult will be to accomplish the valve repair and the plasty will be less stable over time. Some elements as diameter of the LV cavity, the volumes, dilatation of the mitral annulus and migration of the papillary muscle, may be documented by a series of echocardiographic parameters. So, the remodeling of the left heart will be expressed by a series of factor as: tenting area, coaptation distance, left ventricular diameter and volume, the interpapillary distance. Some studies showed negative correlation between these parameters and the feasibility of mitral repair.
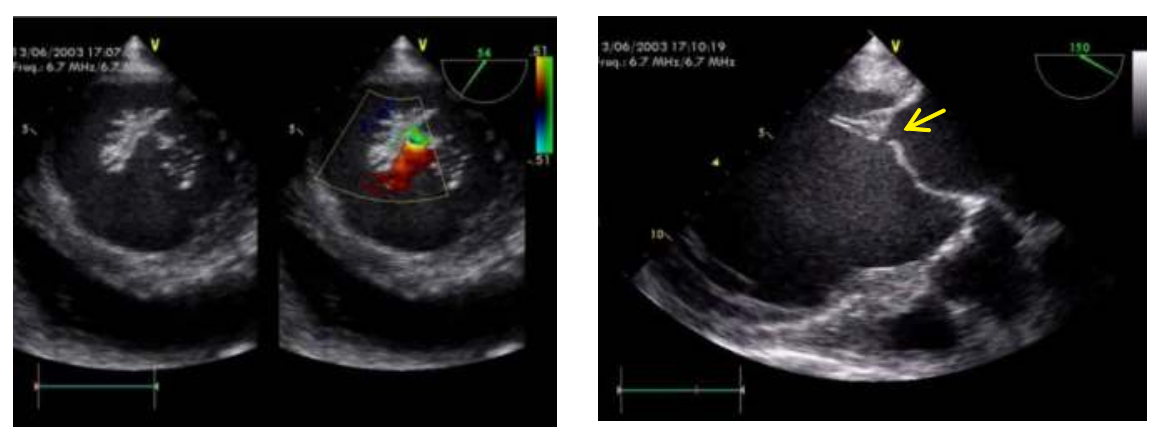

Figure 23. Ischemic dilated cardiomyopathy with chronic mitral regurgitation due to important dilatation of the LV cavity, annular dilatation and loss of leaflet coaptation (arrow). TEE transgastric short and long axis views. Notice also the systolic restriction of the posterior leaflet (right picture). 


\section{Unfavorable characteristics for mitral valve repair (table 2, fig 24)}

When the global remodeling of the left ventricle is too advanced, with loss of the normal shape and important dilatation of the LV cavity, overt tethering of the mitral leaflet and important annular dilatation, the mitral valve repair may be difficult to accomplish (Troubil 2010). A remodeled, dilated ventricle, with an interpapillary muscle distance more than $20 \mathrm{~mm}$, is a marker of poor scenario for mitral valve repair. A left ventricular cavity remodeled and dilated with a value above $65 \mathrm{~mm}$ for $\mathrm{LV}$ end diastolic diameter (EDD) and above $51 \mathrm{~mm}$ for end systolic diameter (ESD), corresponding to an end systolic volume above $140 \mathrm{ml}$, are also considered unfavorable characteristics for valve repair.

- Coaptation distance $\geq 1 \mathrm{~cm}$

- Tenting area $>2,5-3 \mathrm{~cm}^{2}$

- Complex jets

- Posterolateral angle $>45^{\circ}$

- Interpapillary muscle distance $>20 \mathrm{~mm}$

- $\mathrm{EDD}>65 \mathrm{~mm}, \mathrm{ESD}>51 \mathrm{~mm}(\mathrm{ESV}>140 \mathrm{~mL})$

EDD - end diastolic diameter,

ESD - end systolic diameter,

ESV - end systolic volume.

Table 2. Unfavorable characteristics for mitral valve repair.
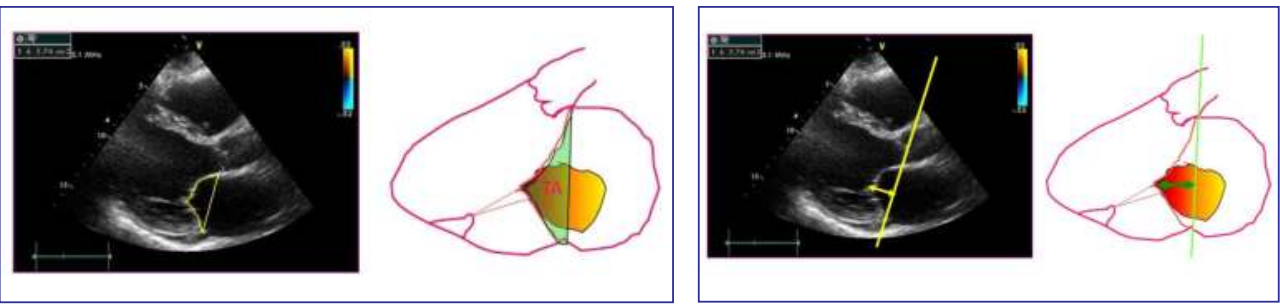

Figure 24. Assessment of the tenting area $\left(5,74 \mathrm{~cm}^{2}\right.$ left) and of the coaptation distance (13mm, right) in a coronary patient with chronic ischemic mitral insufficiency; both criteria suggest low probability for mitral valve repair.

Due to the deteriorated contractile status of the LV, the diminish force of closing contributes to the generation of mitral insufficiency, but this phenomenon alone is not enough. There are indeed forms of dilated cardiomyopathy with very large ventricle and severely reduced ejection fraction without an important mitral regurgitation, or even without any mitral regurgitation. Important in this situation is the impairment of the geometry at the papillary muscles level. Asymmetrical and symmetrical pattern can coexist in the same patient. 
Geometrical echocardiographic analysis. These patients have a high degree of tenting in both leaflets (papillary far from each other), a very dilated annulus and a large defect of coaptation in the central part of the valve conditioning a severe mitral regurgitation, with a large vena contracta. They also present a larger area than normal of the coaptation triangle, express by a larger tenting area and a higher coaptation distance (fig 24).

Surgery. Given the seriousness of this clinical condition, the surgical management of this syndrome requires the most aggressive approach. Restrictive and undersized annuloplasty usually with a complete rigid is often combined with other surgical techniques as leaflet extension and/or papillary muscle repositioning. There are some techniques used in these situations, like re-approaching the papillary muscles with a Gore-Tex band (Hvass 2010) thus decreasing the degree of tenting or the use of an external mesh for preventing further dilatation. Even if the immediate outcome could be satisfactory medium and long term results are to be evaluated.

\section{Chronic ischemic mitral regurgitation due to elongation of papillary muscle (fig 24)}

Background. This type of ischemic mitral regurgitation is considered to be rare (about $2 \%$ of all cases of ischemic mitral regurgitation), belonging to the Carpantier's type II classification. Nevertheless, it is possible that this type of regurgitation is indeed more frequent and in every day practice but also underestimated. The valve leakage is, here, the result of alteration of subvalvular apparatus secondary to ischemic elongation of the papillary muscle following myocardial necrosis, chronic ischemia and mechanical stress. The papillary muscle elongation is never solitary, being an associated process in the general context of postinfarction remodeling.

Mechanism. The partially infarcted and/or ischemic posterior papillary muscle suffers a chronic remodeling with elongation that expresses itself at the level of the mitral leaflets by alteration of the coaptation triangle and valve geometry.

Geometrical echocardiographic analysis: in this specific condition there is an insufficient leaflet coaptation due to various degree of ischemic prolapse of the posterior and/or anterior mitral leaflet. Often the echocardiographic aspect is an unusual anterior leaflet prolapse or that of a prolapse of more corresponding scallops (e.g. $\mathrm{A}_{2} / \mathrm{P}_{2}$ and $\mathrm{A}_{3} / \mathrm{P}_{3}$ ). The papillary muscle appears elongated, triangular shaped, with the tip very close to the mitral annulus. As a consequence, the normal configuration of mitral valve, defined by the coaptation triangle, will suffer various degree of alteration, till the complete disappearance (see also fig 5, 6). An annular dilation is often associated, depending on the extension of myocardial infarction. All these elements will lead to a complex holosystolic regurgitant jet. This entity may be caused by a limited infarction at the papillary muscle level, which lead to fibrosis and elongation of the muscle. The papillary muscle will develop a thin and sharp appearance, like a thorn, and will be more hyperechogenic. 

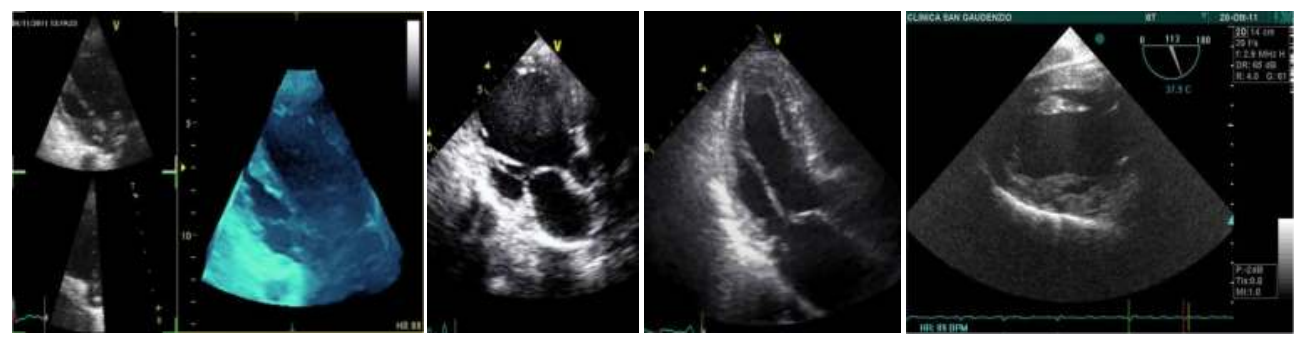

Figure 25. Several patients with chronic ischemic regurgitation and papillary muscle elongation. The papillary muscle appears elongated, thinly and sharply, like a thorn, with a hyperechogenic tip, which is very close to the mitral valve annulus plane (so called "thorny papillary muscle").

The clue of the diagnosis is the visualization of the papillary muscles, which can be done transthoracic in parasternal or apical long axis views, modified for the exposure of the papillary, or transoesophageal at $60^{\circ}$ (bicommissural view) or transgastric view at $90^{\circ}$. The prolapse at the level of one scallop can coexist with the restriction of another scallop, hence the importance to visualize the entire valve prior to surgery.

Surgical planning and management: In these cases a rebuilding of the triangle of coaptation is performed by means of PTFE Gore-Tex neochords which will correct the normal mitral geometry. Depending on the intraoperative finding and surgical exposition and access, plication of the elongated papillary muscle may be performed. Annuloplasty is always performed in order to stabilize the repair.

Dyssynchrony as a cause of chronic ischemic mitral regurgitation. Due to the high prevalence of left bundle branch block in the ischemic heart disease, the assyncronism of contraction is a frequent cause of mitral regurgitation. Significant mitral regurgitation is twice more often in patients with a QRS duration longer than $130 \mathrm{msec}$. The delayed activation of the antero-lateral papillary muscle leads to a misalignment of the leaflets during systole. The resynchronization therapy has good results in this type of mitral regurgitation. The pacing wires are better attached intraoperatively, the surgeon having the possibility to choose the place without the restriction imposed by the anatomy of the cardiac veins.

On one hand, though, the currently available techniques still need validation and, on the other, the optimal therapy /combination of therapies needs clarification.

\section{Conclusions}

Ischemic mitral regurgitation has important prognosis implications in patients with coronary heart disease. It should not be underestimated and this underlines the need for a complete evaluation in which the 2DE and the Doppler echocardiography plays a major role, but should be interpreted specifically, in particular as regards quantification of the regurgitation. 
A complete assessment of left ventricular geometry and function and of the mitral valve configuration prior to surgery may help improve patient risk stratification and better individualize the surgical strategy based on the patient's specific characteristics.

The acute ischemic mitral regurgitation is a rare mechanical complication after myocardial infarction, due to elongation, partial or complete rupture of papillary muscle but has a poor prognosis and it needs urgent diagnosis and surgery. A careful echocardiographic exam, performed in ICU, is mandatory in acute patients with myocardial infarction, focusing the attention on the presence of mitral regurgitation. In case of any suspicion of structural acute ischemic mitral regurgitation the transesophageal exam must be done, to avoid the transformation of partial rupture of papillary muscle in a complete one. The complete rupture of papillary muscle is often fatal and has to be considered a too late clinical and echocardiographic diagnosis; these patients need an emergent operation and very intensive perioperative pharmacologic and mechanical support.

From a conceptual point of view, chronic ischemic mitral regurgitation witnesses the loss of contractile myocardial tissue parallel with the presence of the post-infarction remodeling process. As known, ischemic mitral insufficiency develops on normally mitral leaflets. As the myocardial mass loss is larger, the remodeling processes and regurgitation become greater.

Recognizing the mechanism of valve incompetence is an essential point for the surgical planning and for a good result of the mitral repair. In the patient's candidates to surgery, the role of the echocardiographic exam is to identify the mechanisms of valve regurgitation and to quantify it. As noted, in most cases of chronic ischemic mitral regurgitation the mechanism is the restriction of one or both leaflets, caused by the tethering exercised by the displaced papillary muscle.

The transthoracic exam is commonly enough to clarify the mechanism of the chronic ischemic mitral regurgitation. Only patients with papillary muscle elongation may raise difficulties in diagnosis. Loss of the normal shape of the 'triangle of coaptation' is one of the key characteristics of chronic ischemic mitral regurgitation. In case of post-ischemic elongation of the papillary muscle, the clue of the diagnosis is obtained by looking not only to the mitral valve, but by focusing the exam on the papillary muscles, particularly when the patient presents a deformation of the 'coaptation triangle'. In patients with type $\mathrm{II}^{\circ}$ Carpentier's classifications (elongated papillary muscle and leaflets prolapse), alongside of ring annuloplasty use of PTFE Gore-Tex chordae is often required.

In patients with chronic ischemic mitral insufficiency the echocardiography may identify two different patterns of post infarction remodeling: a limited process of post infarction remodeling and an extensive one. The mitral repair is usually more challenging in patients with extensive pattern of post infarction remodeling, where, due to left heart cavity enlargement a phenomenon of "mitral valve - LV chamber mismatch" is present.

Although the mechanisms of ischemic mitral regurgitation are often complex, currently, undersized annuloplasty is considered the standard approach to reducing mitral insufficiency. Unfortunately this technique does not resolve the real mechanism of disease, the remodeled and sphericalized left ventricle. By this technique the persistence of ischemic mitral regurgi- 
tation is considered to range between 10 to $20 \%$ of the cases, and, at five years of follow up, half of the patient may develop recurrence.

Thus, in advanced stage of left ventricular post infarction remodeling with over dilatation and important mitral valve tethering, the ring annuloplasty has to be completed with leaflet extension. This approach seems coherent with the mechanism of mitral regurgitation, with the echocardiographic data and with the concept of "mitral valve - LV chamber mismatch".

The major challenge for cardiologists and surgeons is how to integrate data from different imaging techniques that assess mitral valve geometry and left ventricular size, geometry, function and potential functional recovery. This in order to tailor and optimize the surgical approach to patients with chronic ischemic mitral regurgitation.

Even today, we have no reliable predictors of recurrent and persistent mitral regurgitation. However, the surgical treatment of ischemic mitral insufficiency continues to evolve and new methods addressing ventricular structures are being introduced: left ventricular restoration, papillary muscle's relocation, sling, imbrication or reapproximation, chordal cutting or translocation. Despite remarkable progress in reparative surgery, further investigation is still necessary to find the best approach to treat ischemic mitral regurgitation.

\section{Author details}

Gheorghe Cerin ${ }^{1}$, Adrian Bogdan Popa ${ }^{1}$, Razvan Ticulescu ${ }^{2}$ and Marco Diena ${ }^{1}$

*Address all correspondence to: gheorghe.cerin@gmail.com

1 The Cardioteam Foundation, Cardiac Surgery Dpt, San Gaudenzio Clinic, Novara, Italy

2 Cardiac Surgery Dpt, The 'Monza' Hospital, Bucharest, Romania

\section{References}

[1] "Kirklin/Barratt-Boyes Cardiac Surgery": Nicholas T. Kouchoukos, Eugene H. Blackstone, Frank L. Hanley, James K. Kirklin. 4Th edition, Publisher: Saunders; 4th Revised edition

[2] Popa B A, Diena M, Cerin G, Lanzillo G., Tesler UF.Severe Post-Ischemic Elongation of the Anterior Papillary Muscle. An Unusual Cause of Mitral Insufficiency in a 75Year-Old Man; Tex Heart Inst J. (2007); 34(2): 222-224.

[3] Carpentier's Reconstructive Valve Surgery, Alain Carpentier, David Adams, FarzanFilsoufi, Saunders, 2010. 
[4] Carpentier A. Cardiac valve surgery:the French correction. J ThoracCardiovascSurg. 1983; 86: 323-337.

[5] Cerin G, Popa BA, Benea D, Lanzillo G, Karazanishvili L, Casati V, Popa A, Novelli E, Renzi L, Diena M. The triangle of coaptation: a new concept to enhance mitral valve repair through reshaping the native geometry. Circ. 2010; 122: e115. Published online before print June 14, 2010, doi: 10.1161/ CIRCULATION AHA.110.192774.

[6] Clements SD Jr, Story WE, Hurst JW, Craver JM, Jones EL. Ruptured papillary muscle, a complication of myocardial infarction: clinical presentation, diagnosis, and treatment. ClinCardiol. (1985);8(2), 93-103.

[7] Cohn LH, Rizzo R J, Adams DH et al. The effect of pathophysiology on the surgical treatment of ischemic mitral regurgitation: operative and late risks of repair versus replacement. Eur J CardioThoracSurg (1995); 9: 568-74.

[8] Dagum P, Timek TA, Green, G R, et al. Coordinate-free analysis of mitral valve dynamics in normal and ischemic hearts. Circulation (2000) 102(19 Suppl 3):III62-9.

[9] De Varennes B, Chaturvedi R, Sidhu S, Côté A. Li Pi Shan W, Goyer C, Hatzakorzian R, Buithieu J, Sniderman A Initial Results of Posterior Leaflet Extension for Severe Type IIIb Ischemic Mitral Regurgitation Circulation. (2009) 2;119(21):2837-43.

[10] Dobre M, Koul B, Rojer A. Anatomic and physiologic correction of the restricted posterior mitral leaflet motion in chronic ischemic mitral regurgitation. J Thor CardiovascSurg. (2000); 120 (2): 409-411.

[11] Estes EH Jr., Dalton FM, Entman ML, Dixon HB II, Hackel DB. The anatomy and blood supply of the papillary muscles of the left ventricle. AmHeart J 1966; 71(3): 356-362.

[12] Fattouch K, Guccione F, Sampognaro R, Panzarella G, Corrado E, Navarra E, Calvaruso D, Ruvolo G. POINT: Efficacy of adding mitral valve restrictive annuloplasty to coronary artery bypass grafting in patients with moderate ischemic mitral valve regurgitation: a randomized trial. J Thorac Cardiovasc Surg. 2009;138:278-85.

[13] Fattouch K, Sampognaro R, Speziale G, Salardino M, Novo G, Caruso M, Novo S, Ruvolo G. Impact of moderate ischemic mitral regurgitation after isolated coronary artery bypass grafting. AnnThoracSurg. 2010;90:1187-94.

[14] Cerin, G, Popa, B A, \&Diena, M. The Degenerative Mitral Valve Regurgitation: From Geometrical Echocardiographic Concepts to Successful Surgical Repair; Echocardiography- In Specific Diseases, (2012). 978-9-53307-977-6DOI:

[15] G Cerin, M Diena, G Lanzillo, S Casalino, A Zito, D Benea, U Filippo Tesler. Degenerative mitral regurgitation - surgical and echocardiographic consideration for repair. Romanian Journal of Cardiovascular Surgery, 5 (3), pp. 131-39, 2006. 
[16] Aklog L, Filsoufi F, Flores KQ, et al: Does coronary artery bypass grafting alone correct moderate ischemic mitral regurgitation? Circulation 2001;104(12 Suppl 1):I68-I75.

[17] Gisbert A, Soulière V, Denault AY, Bouchard D, Couture P, Pellerin M, Carrier M, Levesque S, Ducharme A, Basmadjian AJ. Dynamic quantitative echocardiographic evaluation of mitral regurgitation in the operating department. J AmSocEchocardiogr. 2006 Feb;19(2):140-6.

[18] Green GR, Dagum P, Glasson JR, Daughters GT, Bolger AF, Foppiano LE, Berry GJ, Ingels NB Jr, Miller DC; Mitral annular dilatation and papillary muscle dislocation without mitral regurgitation in sheep. Circulation. 1999 Nov 9;100(19 Suppl):II95-102.

[19] Grigioni F, Enriquez-Sarano, M, Zehr, K. J, Bailey, K. R, \& Tajik, A. J. Ischemic mitral regurgitation: long-term outcome and prognostic implications with quantitative Doppler assessment. Circ. (2001). , 103, 1759-1764.

[20] Grossi EA, Goldberg JD, LaPietra A, Ye X, Zakow P, Sussman M, Delianides J, Culliford AT, Esposito RA, Ribakove GH, Galloway AC, Colvin SB. Ischemic mitral valve reconstruction and replacement: comparison of long-term survival and complications. J ThoracCardiovascSurg. 2001; 122: 1107-1124.

[21] Muresian, H. (2009). The Clinical Anatomy of The Coronary Arteries. An Anatomical Study on Human Heart Specimens. Edited by H. Muresian. Ed. Enciclopedica isbn: 978-973-45-0581-4.

[22] Hvass, U. Joudinaud T The papillary muscle sling for ischemic mitral regurgitation; J Th Cardiovascular Surgery February (2010). , 139(2), 418-423.

[23] Jeroen, J. Bax; Jerry Braun; Soeresh T. Somer, Robert Klautz, Eduard R. Holman, Michel I.M. Versteegh, Eric Boersm, MSc; Martin J. Schalij, Ernst E. van der Wall, Robert A. Dion Restrictive Annuloplasty and Coronary Revascularization in Ischemic Mitral Regurgitation Results in Reverse Left Ventricular Remodeling. Circulation. (2004). suppl II]: II-II-108., 103.

[24] Lamas GA, Mitchell GF, Flaker GC, Smith Jr SC, Gersh BJ, Basta L, Movè L, Braunwald E, Pfeffer MA. Clinical significance of mitral regurgitation after acute myocardial infarction. Survival and Ventricular Enlargement. Circulation 1997; 96:827-833. doi: 10.1161/01.CIR.96.3.827.

[25] Langer F, Rodriguez F, Cheng A, Ortiz S, Nguyen T C, Zasio M K, BA, Liang D, Daughters G T, Ingels N B, D. Miller C. Posterior mitral leaflet extension: An adjunctive repair option for ischemic mitral regurgitation? J Thor CardiovascSurg 2006;131:868-877 .

[26] Levine RA, Hung J, Otsuji Y, Messas E, Liel-Cohen N, Nathan N, Handschumacher MD, Guerrero JL, He S, Yoganathan AP, Vlahakes GJ. Mechanisticinsightsintofunctional mitral regurgitation. Current Cardiol Reports 2002; 4(2):125-129. 
[27] Loisance DY, Deleuze PH, Hillion ML, Cachera JP: Are there indications for reconstructive surgery in severe mitral regurgitation after acute myocardial infarction? Eur J CardiothoracSurg 1990; 4:394-97.

[28] Troubil, M, Lonsky, V, \&Gwozdziewicz, M. V. Bruk Persistent and recurrent ischemic mitral regurgitation; Biomed Pap Med FacUnivPalacky Olomouc Czech Rep. (2010). Jun; 154(2), 141-145.

[29] Magne, J, Sénéchal, M, \&Dumesnil, J. G. Pibarot P; Ischemic mitral regurgitation: a complex multifaceted disease. Cardiology. 2009;doi:Epub (2008). Aug 30, 112(4), 6 244-59.

[30] McGee EC, Gillinov AM, Blackstone EH, Lytle BW, McCarthy PM, Cosgrove DM. Recurrent mitral regurgitation after annuloplasty for functional ischemic mitral regurgitation. J ThoracCardiovascSurg. 2004; 128: 916-924.

[31] Messas E, Guerrero JL, Handschumacher MD, Chow C-M, Sullivan S, Yoganathan A P, Levine RA Chordal cutting: a new therapeutic approach for ischemic mitral regurgitation. Circulation 2001;104(16):1958-1963; doi: 10.1161/hc4201.097135.

[32] Mitesh V. Badiwala, SubodhVerma and VivekRao; Surgical Management of Ischemic Mitral Regurgitation. Circulation 2009; 120; 1287-1293; doi: 10.1161/CIRCULATIONAHA.108.836627.

[33] Monin J-L, Dehant P, Roiron C, Monchi M, Tabet J-Y, Clerc P, Fernandez G, Houel R, Garot J, Chauvel C, Gueret P; Functional assessment of mitral regurgitation by transthoracic echocardiography using standardized imaging planes: diagnostic accuracy and outcome implications. J AmCollCardiol 2005; 46(2):302-309. doi:10.1016/j.jacc. 2005.03.064.

[34] Otto, C. (2012). The Practice of Clinical Echocardiography, 4th Edition, W. B. Saunders Company, 978-1-43772-765-4Philadelphia, USA.

[35] Patrick Klein. Jerry Braun, Eduard R. Holman, Michel I.M. Versteegh, Harriette F. Verwey, Robert A.E. Dion, Jeroen J. Bax and Robert J.M. Klautz; Management of mitral regurgitation during left ventricular reconstruction for Iischemic heart failure European Journal of Cardio-Thoracic Surgery (2012). doi:10.1016/j.ejcts.2011.04.035, 41(2012), 74-81.

[36] Zegdi R, Khabbaz Z, Chauvaud S, Latremouille C, Fabiani J-N, \&Deloche A. Posterior Leaflet Extension With an Autologous Pericardial Patch in Rheumatic Mitral Insufficiency Ann ThoracSurg (2007), 84, 1043-4.

[37] Rankin JS, Feneley MP, Hickey M St J, Muhlbaier LH, Wechsler AS, Floyd RA, Skelton TN, Califf RM, Lowe JE et al. A clinical comparison of mitral valve repair versus valve replacement in ischemic mitral regurgitation. J ThoracCardiovascSurg 1988;95:165-77.

[38] Stevenson L. W. Grover-McKay M, Brucken RC, et al: Effects of afterload reduction (diuretics and vasodilators) on left ventricular volume and mitral regurgitation in se- 
vere congestive heart failure secondary to ischemic or idiopathic dilated cardiomyopathy. Am J Cardiol (1987); 60, 654-658.

[39] Tcheng JE, Jackman JD Jr, Nelson CL, gardner LH, Rankin JS, Califf RM, Stack RS: Outcome of patients sustaining acute ischemic mitral regurgitation during myocardial infarction. AnnIntMed 1992; 117:18-24.

[40] Timek TA, Lai DT, Tibayan F, Liang D, Daughters GT, Dagum P, Zasio MK, Lo S, Hastie T, Ingles NB Jr, Miller DC. Ischemia in three left ventricular regions: Insights into the pathogenesis of acute ischemic mitral regurgitation. J ThoracCardiovascSurg 2003;125(3):559-569.

[41] Tesler, U F, Cerin, G, \& Novelli, E. Anamaria Popa, M Diena. Evolution of SurgicalTechniques for Mitral Valve Repair. Tex HeartInst J. (2009). PMCID: PMC2763455, 36(5), 438-440.

[42] Zoghbi WA, Enriquez-Sarano M, Foster E, et al. Recommendations for the evaluation of the severity of native valvular regurgitation with two-dimensional and Doppler echocardiography. J AmSoc of Echocardiography. 2003;16:777-80.

[43] Hashim SW, Yousuef SJ, Ayash B, Rousou AJ, Ragnarsson S, ColazzoS,Geirson A, Pseudoprolpase of the anterior leaflet in chronic mitral ischemic regurgitation: Identification and repair. J ThoraciccardiovascSurg 2012, 143; S33-S37. 
